九州大学学術情報リポジトリ

Kyushu University Institutional Repository

\title{
AN EDGEWORTH EXPANSION OF A CONVEX COMBINATION OF U-STATISTICS BASED ON STUDENTIZATION
}

Yamato, Hajime

Department of Mathematics and Computer Science, Kagoshima Univ.

Toda, Koichiro

Kagoshima Koto Preparatory School

Nomachi, Toshifumi

Miyakonojo College of Technology

Maesono, Yoshihiko

Faculty of Economics, Kyushu University

https://doi.org/10.5109/12582

出版情報: Bulletin of informatics and cybernetics. 36, pp.105-130，2004-12. Research Association of Statistical Sciences

バージョン :

権利関係 : 


\title{
AN EDGEWORTH EXPANSION OF A CONVEX COMBINATION OF U-STATISTICS BASED ON STUDENTIZATION
}

\author{
by \\ Hajime Yamato, Koichiro Toda, Toshifumi Nomachi \\ and \\ Yoshihiko MaEsono
}

Reprinted from the Bulletin of Informatics and Cybernetics

Research Association of Statistical Sciences, Vol.36
FUKUOKA, JAPAN
2004




\title{
AN EDGEWORTH EXPANSION OF A CONVEX COMBINATION OF U-STATISTICS BASED ON STUDENTIZATION
}

\author{
By \\ Hajime Yamato, Koichiro Toda $^{\dagger} \quad$ Toshifumi NomaChI ${ }^{\ddagger}$ \\ and \\ Yoshihiko MAESONo ${ }^{\S}$
}

\begin{abstract}
As an estimator of an estimable parameter, Toda and Yamato (2001) introduce Y-statistic which is a convex combination of U-statistics including V-statistic and LB-statistic. We give the Edgeworth expansions of studentized Y-statistic about the estimable parameter using a jackknife variance estimator, with remainder $o\left(n^{-1}\right)$.
\end{abstract}

Key Words and Phrases: Edgeworth expansion, Convex combination of U-statistics, Studentization.

\section{Introduction}

Let $\theta(F)$ be an estimable parameter of an unknown distribution $F$. Let $g\left(x_{1}, \ldots, x_{k}\right)$ be the symmetric kernel of degree $k(\geq 2)$ for this parameter $\theta(F)$. In this paper, we assume that the kernel $g$ is not degenerate. Let $X_{1}, \ldots, X_{n}$ be a random sample of size $n$ from the distribution $F$. Let $X$ be a random variable having the distribution $F$.

As an estimator of $\theta(F)$, a convex combination $Y_{n}$ of U-statistics is introduced by Toda and Yamato (2001) as follows: Let $w\left(r_{1}, \ldots, r_{j} ; k\right)$ be a nonnegative and symmetric function of positive integers $r_{1}, \ldots, r_{j}$ such that $j=1, \ldots, k$ and $r_{1}+\cdots+r_{j}=k$, where $k$ is the degree of the kernel $g$ and fixed. We assume that at least one of $w\left(r_{1}, \ldots, r_{j} ; k\right)$ 's is positive. For $j=1, \ldots, k$, let $g_{(j)}\left(x_{1}, \ldots, x_{j}\right)$ be the kernel given by

$$
g_{(j)}\left(x_{1}, \ldots, x_{j}\right)=\frac{1}{d(k, j)} \sum_{r_{1}+\cdots+r_{j}=k}^{+} w\left(r_{1}, \ldots, r_{j} ; k\right) g(\underbrace{x_{1}, \ldots, x_{1}}_{r_{1}}, \ldots, \underbrace{x_{j}, \ldots, x_{j}}_{r_{j}}),
$$

where the summation $\sum_{r_{1}+\cdots+r_{j}=k}^{+}$is taken over all positive integers $r_{1}, \ldots, r_{j}$ satisfying $r_{1}+\cdots+r_{j}=k$ with $j$ and $k$ fixed and $d(k, j)=\sum_{r_{1}+\cdots+r_{j}=k}^{+} w\left(r_{1}, \ldots, r_{j} ; k\right)$ for $j=1,2, \ldots, k$. Let $U_{n}^{(j)}$ be the U-statistic associated with this kernel $g_{(j)}\left(x_{1}, \ldots, x_{j}\right)$

\footnotetext{
* Department of Mathematics and Computer Science, Kagoshima Univ., Kagoshima 890-0065, Japan. yamato@sci.kagoshima-u.ac.jp

† Kagoshima Koto Preparatory School, Kagoshima 890-0051, Japan.

¥ Miyakonojo College of Technology, Miyakonojo, Miyazaki 885-8567, Japan.

$\S$ Faculty of Economics, Kyushu University 27, Hakozaki 6-19-1, Higasi-ku, Fukuoka 812-8581, Japan.
} 
for $j=1, \ldots, k$. The kernel $g_{(j)}\left(x_{1}, \ldots, x_{j}\right)$ is symmetric because of the symmetry of $w\left(r_{1}, \ldots, r_{j} ; k\right)$. If $d(k, j)$ is equal to zero for some $j$, then the associated $w\left(r_{1}, \ldots, r_{j} ; k\right)$ 's are equal to zero. In this case, we let the corresponding statistic $U_{n}^{(j)}$ be zero. The statistic $Y_{n}$ is given by

$$
Y_{n}=\frac{1}{D(n, k)} \sum_{j=1}^{k} d(k, j)\left(\begin{array}{c}
n \\
j
\end{array}\right) U_{n}^{(j)},
$$

where $D(n, k)=\sum_{j=1}^{k} d(k, j)\left(\begin{array}{c}n \\ j\end{array}\right)$. Since $w$ 's are nonnegative and at least one of them is positive, $D(n, k)$ is positive. Note that $U_{n}^{(k)}$ is equal to the U-statistic $U_{n}$ given below for $w(1, \ldots, 1 ; k)>0$, because of $g_{(k)}=g$.

Another type of a linear combination of U-statistics, $L_{n}$, is introduced by (3.3) of Sen (1977). While $Y_{n}$ and $L_{n}$ are both linear combination of U-statistics, $Y_{n}$ is different from $L_{n}$ in the mean that the weight function $w$ 's determines $Y_{n}$ as an estimator of $\theta$. Since the coefficients of $U_{n}^{(\cdot)}$ on the right-hand side of (1.2) are non-negative and their sum is equal to one, the linear combination given by (1.2) is also a convex combination.

For example, let $w$ be the function given by $w(1,1, \ldots, 1 ; k)=1$ and $w\left(r_{1}, \ldots, r_{j} ; k\right)$ $=0$ for positive integers $r_{1}, \ldots, r_{j}$ such that $j=1, \ldots, k-1$ and $r_{1}+\cdots+r_{j}=$ $k$. Then the corresponding statistic $Y_{n}$ is equal to U-statistic $U_{n}$, which is given by $U_{n}=\left(\begin{array}{l}n \\ k\end{array}\right)^{-1} \sum_{1 \leq j_{1}<\cdots<j_{k} \leq n} g\left(X_{j_{1}}, \ldots, X_{j_{k}}\right)$, where $\sum_{1 \leq j_{1}<\cdots<j_{k} \leq n}$ denotes the summation over all integers $j_{1}, \ldots, j_{k}$ satisfying $1 \leq j_{1}<\cdots<j_{k} \leq n$.

Let $w$ be the function given by $w\left(r_{1}, \ldots, r_{j} ; k\right)=1$ for positive integers $r_{1}, \ldots, r_{j}$ such that $j=1, \ldots, k$ and $r_{1}+\cdots+r_{j}=k$. Then the corresponding statistic $Y_{n}$ is equal to the LB-statistic $B_{n}$ given by $B_{n}=\left(\begin{array}{c}n+k-1 \\ k\end{array}\right)^{-1} \sum_{r_{1}+\cdots+r_{n}=k} g\left(X_{1}, \ldots, X_{1}, \ldots, X_{n}, \ldots\right.$, $\left.X_{n}\right)$, where the numbers of $X_{1}, \ldots, X_{n}$ are $r_{1}, \ldots, r_{n}$, respectively, and $\sum_{r_{1}+\cdots+r_{n}=k}$ denotes the summation over all non-negative integers $r_{1}, \ldots, r_{n}$ satisfying $r_{1}+\cdots+r_{n}=k$.

Let $w$ be the function given by $w\left(r_{1}, \ldots, r_{j} ; k\right)=k ! /\left(r_{1} ! \cdots r_{j} !\right)$ for positive integers $r_{1}, \ldots, r_{j}$ such that $j=1, \ldots, k$ and $r_{1}+\cdots+r_{j}=k$. Then the corresponding statistic $Y_{n}$ is equal to the V-statistic $V_{n}$ given by $V_{n}=n^{-k} \sum_{j_{1}=1}^{n} \cdots \sum_{j_{k}=1}^{n} g\left(X_{j_{1}}, \ldots, X_{j_{k}}\right)$. (See Toda and Yamato (2001).)

Let $w$ be the function given by $w\left(r_{1}, \ldots, r_{j} ; k\right)=k ! /\left(r_{1} \cdots r_{j}\right)$ for positive integers $r_{1}, \ldots, r_{j}$ such that $j=1, \ldots, k$ and $r_{1}+\cdots+r_{j}=k$. Then, for example, the corresponding statistic $Y_{n}$ for the third central moment of the distribution $F$ is given by $S_{n}=n\left(n^{2}+1\right)^{-1} \sum_{i=1}^{n}\left(X_{i}-\bar{X}\right)^{3}$, where $\bar{X}$ is the sample mean of $X_{1}, \ldots, X_{n}$ (see Nomachi et al. (2002)).

The Edgeworth expansion of the standardized Y-statistic $Y_{n}$ about $\theta$ is obtained with remainder $o\left(n^{-1}\right)$ by Yamato et al. (2003). It also gives the Edgeworth expansion of the studentized Y-statistic with remainder $o\left(n^{-1 / 2}\right)$. For the studentization of $\mathrm{Y}$ statistic $Y_{n}$ given by (1.2), we use a jackknife variance estimator. That is, as a variance 
estimator of $\sqrt{n} Y_{n}$, we use $\hat{\sigma}_{n}^{2}$ given by

$$
\hat{\sigma}_{n}^{2}=(n-1) \sum_{i=1}^{n}\left(Y_{n}^{(i)}-Y_{n}\right)^{2}
$$

where $Y_{n}^{(i)}$ is the Y-statistic given by (1.2) computed from a sample of size $n-1$ with $X_{i}$ left out.

Our purpose is to get an Edgeworth expansion of the studentized statistic $Y_{n}$ given by (1.2), using the jackknife variance estimator $\hat{\sigma}_{n}^{2}$ with remainder term $o\left(n^{-1}\right)$. For the studentized U-statistic, Helmers (1991) and Maesono (1995) obtained its Edgeworth expansion using a jackknife variance estimator with remainder term $o\left(n^{-1 / 2}\right)$. Maesono (1997) get an Edgeworth expansion using a jackknife variance estimator with remainder term $o\left(n^{-1}\right)$. Maesono (1996) gave an Edgeworth expansion of $\sqrt{n}\left[L_{n}-E\left(L_{n}\right)\right] / \tilde{\sigma}_{n}$, where $\tilde{\sigma}_{n}^{2}$ is a jackknife variance estimator of $\sqrt{n}\left[L_{n}-E\left(L_{n}\right)\right]$.

In Section 2, we give an Edgeworth expansion of $\sqrt{n}\left[Y_{n}-E\left(Y_{n}\right)\right] / \hat{\sigma}_{n}$, following Maesono (1996). In Section 3, using the result of Section 2 we shall derive another Edgeworth expansion about parameter $\theta$, that is, the expansion of $\sqrt{n}\left[Y_{n}-\theta\right] / \hat{\sigma}_{n}$. We give some examples in Section 4 . In Section 5 , we give supplementary propositions necessary for the previous sections.

\section{Studentized Y-statistic about its expectation}

In the following sections, we assume $d(k, k)>0$. Then, with $\delta_{k}=k d(k, k-$ $1) / d(k, k)$ it holds that

$$
\frac{d(k, k)}{D(n, k)}\left(\begin{array}{l}
n \\
k
\end{array}\right)=1-\frac{\delta_{k}}{n}+O\left(\frac{1}{n^{2}}\right)
$$

and

$$
\frac{d(k, k-1)}{D(n, k)}\left(\begin{array}{c}
n \\
k-1
\end{array}\right)=\frac{\delta_{k}}{n}+O\left(\frac{1}{n^{2}}\right) .
$$

For the U-statistic $U_{n}, d(k, k) n^{(k)} /[D(n, k) k !]=1$ and $\delta_{k}=0$. For the V-statistic $V_{n}$ and the S-statistic $S_{n}, \delta_{k}=k(k-1) / 2$. For the LB-statistic $B_{n}, \delta_{k}=k(k-1)$ (see Nomachi et al. (2002)).

We put

$$
\psi_{c}\left(x_{1}, \ldots, x_{c}\right)=E\left[g\left(X_{1}, \ldots, X_{k}\right) \mid X_{1}=x_{1}, \ldots, X_{c}=x_{c}\right], \quad c=1,2,3
$$

and

$$
g^{(1)}\left(x_{1}\right)=\psi_{1}\left(x_{1}\right)-\theta,
$$

for $c=2,3$

$$
g^{(c)}\left(x_{1}, \ldots, x_{c}\right)=\psi_{c}\left(x_{1}, \ldots, x_{c}\right)-\sum_{i=1}^{c-1} \sum_{1 \leq l_{1}<\cdots<l_{i} \leq c} g^{(i)}\left(x_{l_{1}}, \ldots, x_{l_{i}}\right)-\theta .
$$


For the kernel $g_{(k-1)}\left(x_{1}, \ldots, x_{k-1}\right)$, we put

$$
\begin{gathered}
\theta_{k-1}=E g_{(j)}\left(X_{1}, \ldots, X_{k-1}\right), \\
\psi_{(k-1), 1}\left(x_{1}\right)=E\left[g_{(k-1)}\left(X_{1}, \ldots, X_{k-1}\right) \mid X_{1}=x_{1}\right],
\end{gathered}
$$

and

$$
g_{(k-1)}^{(1)}\left(x_{1}\right)=\psi_{(k-1), 1}\left(x_{1}\right)-\theta_{k-1} .
$$

We put

$$
\begin{aligned}
\sigma_{1}^{2} & =E\left[\left\{g^{(1)}(X)\right\}^{2}\right], \quad \sigma_{2}^{2}=(k-1)^{2} E\left[\left\{g^{(2)}\left(X_{1}, X_{2}\right)\right\}^{2}\right], \\
\nu & =\sigma_{2}^{2}+\frac{2(k-1) \delta_{k}}{k} E\left[g^{(1)}(X) g_{(k-1)}^{(1)}(X)\right]-2 \delta_{k} \sigma_{1}^{2}, \\
f_{1}(x) & =\frac{1}{2}\left[\left\{g^{(1)}(x)\right\}^{2}-\sigma_{1}^{2}\right]+(k-1) E\left[g^{(1)}\left(X_{2}\right) g^{(2)}\left(x, X_{2}\right)\right]
\end{aligned}
$$

and

$$
\begin{aligned}
f_{2}(x, y)= & -g^{(1)}(x) g^{(1)}(y)+(k-1)\left\{g^{(2)}(x, y)\left[g_{1}(x)+g_{1}(y)\right]\right. \\
& \left.-E\left[g^{(2)}\left(x, X_{3}\right) g^{(1)}\left(X_{3}\right)\right]-E\left[g^{(2)}\left(y, X_{3}\right) g^{(1)}\left(X_{3}\right)\right]\right\} \\
& +(k-1)^{2} E\left[g^{(2)}\left(x, X_{3}\right) g^{(2)}\left(y, X_{3}\right)\right] \\
& +(k-1)(k-2) E\left[g^{(3)}\left(x, y, X_{3}\right) g^{(1)}\left(X_{3}\right)\right],
\end{aligned}
$$

which satisfy the relations $E f_{1}(X)=0$ and $E\left[f_{2}\left(X_{1}, X_{2}\right) \mid X_{1}\right]=0$ a.s. (almost surely), respectively. Furthermore we put

$$
\begin{aligned}
\tau & =\frac{3 E\left[f_{1}^{2}\left(X_{1}\right)\right]}{2 \sigma_{1}^{4}}-\frac{\nu}{2 \sigma_{1}^{2}}, \\
\zeta & =E\left[f_{1}\left(X_{1}\right) g^{(1)}\left(X_{1}\right)\right]
\end{aligned}
$$

and

$$
\begin{aligned}
a_{1}(x)= & \frac{\delta_{k}}{k}\left[(k-1) g_{(k-1)}^{(1)}(x)-k g^{(1)}(x)\right]+\tau g^{(1)}(x) \\
& -\frac{1}{\sigma_{1}^{2}}\left\{\left[f_{1}(x) g^{(1)}(x)-\zeta\right]+\left(E\left[f_{2}\left(x, X_{2}\right) g^{(1)}\left(X_{2}\right)\right]-\frac{3 \zeta}{\sigma_{1}^{2}} f_{1}(x)\right)\right. \\
& \left.+(k-1) E\left[g^{(2)}\left(x, X_{2}\right) f_{1}\left(X_{2}\right)\right]\right\}, \\
a_{2}(x, y)= & (k-1) g^{(2)}(x, y)-\frac{1}{\sigma_{1}^{2}}\left[f_{1}(x) g^{(1)}(y)+f_{1}(y) g^{(1)}(x)\right], \\
a_{3}(x, y, z)= & (k-1)(k-2) g^{(3)}(x, y, z) \\
& -\frac{1}{\sigma_{1}^{2}}\left\{(k-1)\left[f_{1}(x) g^{(2)}(y, z)+f_{1}(y) g^{(2)}(x, z)+f_{1}(z) g^{(1)}(x, y)\right]\right. \\
& +g^{(1)}(x)\left[f_{2}(y, z)-\frac{3}{\sigma_{1}^{2}} f_{1}(y) f_{1}(z)\right]+g^{(1)}(y)\left[f_{2}(x, z)-\frac{3}{\sigma_{1}^{2}} f_{1}(x) f_{1}(z)\right] \\
& \left.+g^{(1)}(z)\left[f_{2}(x, y)-\frac{3}{\sigma_{1}^{2}} f_{1}(x) f_{1}(y)\right]\right\}
\end{aligned}
$$


which satisfy the relations $E\left[a_{1}(X)\right]=0, E\left[a_{2}\left(X_{1}, X_{2}\right) \mid X_{1}\right]=E\left[a_{2}\left(X_{1}, X_{2}\right) \mid X_{2}\right]=0$ and $E\left[a_{3}\left(X_{1}, X_{2}, X_{3}\right) \mid X_{1}, X_{2}\right]=0$ a.s. because of $E f_{1}(X)=0, E\left[f_{2}\left(X_{1}, X_{2}\right) \mid X_{1}\right]=0$ and $E\left[g^{(2)}\left(X_{1}, X_{2}\right) \mid X_{2}\right]=0$ a.s. We define

$$
\begin{aligned}
& \lambda_{1}=E\left[g^{(1)}\left(X_{1}\right)\right]^{3}, \\
& \lambda_{2}=E\left[g^{(1)}\left(X_{1}\right) g^{(1)}\left(X_{2}\right) g^{(2)}\left(X_{1}, X_{2}\right)\right], \\
& \lambda_{3}=E\left[g^{(1)}\left(X_{1}\right)\right]^{4}, \\
& \lambda_{4}=E\left[\left(g^{(1)}\left(X_{1}\right)\right)^{2} g^{(1)}\left(X_{2}\right) g^{(2)}\left(X_{1}, X_{2}\right)\right], \\
& \lambda_{5}=E\left[g^{(1)}\left(X_{1}\right) g^{(1)}\left(X_{2}\right) g^{(2)}\left(X_{1}, X_{3}\right) g^{(2)}\left(X_{2}, X_{3}\right)\right], \\
& \lambda_{6}=E\left[g^{(1)}\left(X_{1}\right) g^{(1)}\left(X_{2}\right) g^{(1)}\left(X_{3}\right) g^{(3)}\left(X_{1}, X_{2}, X_{3}\right)\right], \\
& \lambda_{7}=E\left[g^{(1)}\left(X_{1}\right) a_{1}\left(X_{1}\right)\right], \\
& \kappa_{3}=\sigma_{1}^{-3}\left(\lambda_{1}+3 \lambda_{2}\right), \\
& \kappa_{4}=\sigma_{1}^{-4}\left(\lambda_{3}-3 \sigma_{1}^{4}+12 \lambda_{4}+12 \lambda_{5}+4 \lambda_{6}\right)
\end{aligned}
$$

and

$$
\begin{aligned}
Q_{n}(x)= & \Phi(x)-\phi(x)\left\{\frac{\kappa_{3}}{6 \sqrt{n}}\left(x^{2}-1\right)+\frac{\kappa_{4}}{24 n}\left(x^{3}-3 x\right)\right. \\
& \left.+\frac{\kappa_{3}^{2}}{72 n}\left(x^{5}-10 x^{3}+15 x\right)+\frac{x}{n \sigma_{1}^{2}}\left(\lambda_{7}+\frac{1}{4} E\left[a_{2}^{2}\left(X_{1}, X_{2}\right)\right]\right)\right\} .
\end{aligned}
$$

Lemma 2.1. (Maesono (1996)) If $E\left|g\left(X_{i_{1}}, \ldots, X_{i_{k}}\right)\right|^{2}<\infty$ for $1 \leq i_{1} \leq \cdots \leq i_{k} \leq$ $k$, and $E\left|g\left(X_{1}, X_{2}, X_{3}, \ldots, X_{k}\right)\right|^{4+\varepsilon}<\infty$ and $E\left|g\left(X_{1}, X_{1}, X_{2}, \ldots, X_{k}\right)\right|^{4+\varepsilon}<\infty$ for $\varepsilon>0$, then we have

$$
\hat{\sigma}_{n}^{2}=k^{2} \sigma_{1}^{2}+\frac{2 k^{2}}{n} \sum_{i=1}^{n} f_{1}\left(X_{i}\right)+\frac{2 k^{2}}{n(n-1)} \sum_{1 \leq i<j \leq n} f_{2}\left(X_{i}, X_{j}\right)+\frac{k^{2} \nu}{n}+o_{p}^{*}\left(n^{-1}\right)
$$

and

$$
\begin{array}{r}
k \sigma_{1} \hat{\sigma}_{n}^{-1}=1-\frac{1}{n \sigma_{1}^{2}} \sum_{i=1}^{n} f_{1}\left(X_{i}\right)-\frac{1}{n^{2} \sigma_{1}^{2}} \sum_{1 \leq i<j \leq n}\left[f_{2}\left(X_{i}, X_{j}\right)-\frac{3}{\sigma_{1}^{2}} f_{1}\left(X_{i}\right) f_{1}\left(X_{j}\right)\right] \\
+\frac{1}{n}\left\{\frac{3 E\left[f_{1}^{2}\left(X_{1}\right)\right]}{2 \sigma_{1}^{4}}-\frac{\nu}{2 \sigma_{1}^{2}}\right\}+o_{p}^{*}\left(n^{-1}\right)
\end{array}
$$

where $o_{p}^{*}\left(n^{-1}\right)$ is a quantity satisfying $P\left(\left|o_{p}^{*}\left(n^{-1}\right)\right| \geq c n^{-1}(\log n)^{-1}\right)=o\left(n^{-1}\right)$ for $a$ constant $c>0$.

Thus by Maesono (1996) we have the following: Assume that $E\left|g\left(X_{i_{1}}, \ldots, X_{i_{k}}\right)\right|^{2}<$ $\infty$ for $1 \leq i_{1} \leq \cdots \leq i_{k} \leq k, E\left|g\left(X_{1}, X_{2}, X_{3}, \ldots, X_{k}\right)\right|^{9}<\infty$ and $E \mid g\left(X_{1}, X_{1}, X_{2}, \ldots\right.$, $\left.X_{k}\right)\left.\right|^{4+\varepsilon}<\infty$ for $\varepsilon>0$. Then we have

$$
\hat{\sigma}_{n}^{-1} \sqrt{n}\left(Y_{n}-E\left[Y_{n}\right]\right)=\frac{\sqrt{n}}{\sigma_{1}} U_{n}^{*}-\frac{\zeta}{\sqrt{n} \sigma_{1}^{3}}+o_{p}^{*}\left(n^{-1}\right),
$$


where

$U_{n}^{*}=\frac{1}{n} \sum_{i=1}^{n}\left\{g^{(1)}\left(X_{i}\right)+\frac{a_{1}\left(X_{i}\right)}{n}\right\}+\frac{1}{n^{2}} \sum_{1 \leq i<j \leq n} a_{2}\left(X_{i}, X_{j}\right)+\frac{1}{n^{3}} \sum_{1 \leq i<j<l \leq n} a_{3}\left(X_{i}, X_{j}, X_{l}\right)$.

For Edgeworth expansion of the statistic, we use the result of Lai and Wang (1993) which needs the following conditions.

Condition (C): $E\left|g^{(2)}\right|^{r}<\infty$ for some $r>2$ and there exists $K$ Borel functions $h_{j}: R \rightarrow R$ such that $K(r-2)>8(4 r-5), E h_{j}^{2}\left(X_{1}\right)<\infty(j=1, \ldots, K)$, and the covariance matrix of $\left(W_{1}, \ldots, W_{K}\right)$ is positive definite, where $W_{j}=\left(L h_{j}\right)\left(X_{1}\right)$ and $(L h)(x)=E\left[a_{2}\left(x, X_{2}\right) h\left(X_{2}\right)\right]$.

In case of $k \geq 3$, the original condition of Lai and Wang (1993) contains the term $I_{\left[E\left|g^{(3)}\left(X_{1}, X_{2}, X_{3}\right)\right|>0\right]}$, which equals 1 since $g^{(3)}\left(X_{1}, X_{2}, X_{3}\right)$ is not zero a.s. under the assumption that the kernel $g$ is not degenerate, that is, $\sigma_{1}^{2}>0$. It also contains $E\left|g^{(2)}\right|^{r}<\infty$ for some $r>2$. This condition is satisfied with $r=4$ under our condition $E\left[\left|\psi_{3}\left(X_{1}, X_{2}, X_{3}\right)\right|^{4}\right]<\infty$, which is necessary for the condition (A4) of Lai and Wang (1993).

Condition (D): There exist constants $c_{j}$ and Borel functions $h_{j}: R \rightarrow R$ such that $E h_{j}\left(X_{1}\right)=0, E\left|h_{j}\left(X_{1}\right)\right|^{r}<\infty$ for some $r \geq 5$ and $a_{2}\left(X_{1}, X_{2}\right)=\sum_{j=1}^{K} c_{j} h_{j}\left(X_{1}\right) h_{j}\left(X_{2}\right)$ a.s.; moreover, for some $0<\varepsilon<\min \left\{1,2\left(1-11 r^{-1} / 3\right)\right\}$,

$$
\limsup _{|t| \rightarrow \infty} \sup _{\left|s_{1}\right|+\cdots+\left|s_{K}\right| \leq|t|^{-\varepsilon}}\left|E \exp \left(i t\left[g^{(1)}\left(X_{1}\right)+\sum_{j=1}^{K} s_{j} h_{j}\left(X_{1}\right)\right]\right)\right|<1
$$

The asymptotic expansion of the statistic $\sqrt{n} \sigma_{1}^{-1} U_{n}^{*}$ is given by the following.

Lemma 2.2. (Maesono (1996)) Assume that $\left.E\left[g^{(1)}\left(X_{1}\right)\right)\right]^{4}<\infty, \sigma_{1}^{2}>0$, $E\left[\left|a_{1}\left(X_{1}\right)\right|^{3}+\left|a_{3}\left(X_{1}, X_{2}, X_{3}\right)\right|^{4}\right]<\infty$ and $\lim \sup _{|t| \rightarrow \infty}\left|E\left[\exp \left\{i t g^{(1)}\left(X_{1}\right)\right\}\right]\right|<1$. If either condition $(\mathrm{C})$ or $(\mathrm{D})$ is satisfied, we have

$$
\sup _{-\infty<x<\infty}\left|P\left(\sqrt{n} \sigma_{1}^{-1} U_{n}^{*} \leq x\right)-Q_{n}(x)\right|=o\left(n^{-1}\right) .
$$

For example, by Minkowski's inequality and Schwarz's one, one of the above conditions $E\left[\left|a_{1}\left(X_{1}\right)\right|^{3}+\left|a_{3}\left(X_{1}, X_{2}, X_{3}\right)\right|^{4}\right]<\infty$ is satisfied if

$$
\begin{gathered}
E\left|g^{(1)}\left(X_{1}\right)\right|^{12}<\infty, \quad E\left|g^{(2)}\left(X_{1}, X_{2}\right)\right|^{12}<\infty, \\
E\left|g^{(3)}\left(X_{1}, X_{2}, X_{3}\right)\right|^{12}<\infty, \quad E\left|g_{(k-1)}^{(1)}\left(X_{1}\right)\right|^{3}<\infty .
\end{gathered}
$$


We define

$$
\begin{aligned}
& e_{1}=E\left[g^{(1)}\left(X_{1}\right)\right]^{3}, \\
& e_{2}=(k-1) E\left[g^{(1)}\left(X_{1}\right) g^{(1)}\left(X_{2}\right) g^{(2)}\left(X_{1}, X_{2}\right)\right], \\
& e_{3}=E\left[g^{(1)}\left(X_{1}\right)\right]^{4}, \\
& e_{4}=(k-1) E\left[\left(g^{(1)}\left(X_{1}\right)\right)^{2} g^{(1)}\left(X_{2}\right) g^{(2)}\left(X_{1}, X_{2}\right)\right], \\
& e_{5}=(k-1)^{2} E\left[g^{(1)}\left(X_{1}\right) g^{(1)}\left(X_{2}\right) g^{(2)}\left(X_{1}, X_{3}\right) g^{(2)}\left(X_{2}, X_{3}\right)\right], \\
& e_{6}=(k-1)(k-2) E\left[g^{(1)}\left(X_{1}\right) g^{(1)}\left(X_{2}\right) g^{(1)}\left(X_{3}\right) g^{(3)}\left(X_{1}, X_{2}, X_{3}\right)\right], \\
& v_{1}=\sigma_{1}^{-3}\left(2 e_{1}+3 e_{2}\right), \\
& v_{2}=\sigma_{1}^{-3}\left(e_{1}+3 e_{2}\right), \\
& v_{3}=-\sigma_{1}^{-6}\left(2 e_{1}+3 e_{2}\right)^{2}, \\
& v_{4}=6 \sigma_{1}^{-4}\left(e_{3}-6 \sigma_{1}^{4}+12 e_{4}+6 e_{5}+4 e_{6}\right)-2 \sigma_{1}^{-6}\left(2 e_{1}+3 e_{2}\right)\left(2 e_{1}+9 e_{2}\right), \\
& v_{5}=3 \sigma_{1}^{-6}\left(4 e_{1}^{2}+12 e_{1} e_{2}+3 e_{2}^{2}\right)+18 \sigma_{1}^{-4}\left(\sigma_{1}^{2} \sigma_{2}^{2}-e_{3}+2 \sigma_{1}^{4}-4 e_{4}-2 e_{5}\right) .
\end{aligned}
$$

Using $f_{12}(x)=E\left[g^{(1)}\left(X_{2}\right) g^{(2)}\left(x, X_{2}\right)\right]$ which appears in the second term of $f_{1}$, we can write

$$
\begin{gathered}
e_{2}=(k-1) E\left[g^{(1)}\left(X_{1}\right) f_{12}\left(X_{1}\right)\right], \quad e_{4}=(k-1) E\left[\left\{g^{(1)}\left(X_{1}\right)\right\}^{2} f_{12}\left(X_{1}\right)\right], \\
e_{5}=(k-1)^{2} E\left[\left\{f_{12}\left(X_{1}\right)\right\}^{2}\right] .
\end{gathered}
$$

Furthermore, we define

$$
H_{n}(x)=\Phi(x)+\phi(x) \frac{1}{6 \sqrt{n}}\left(v_{1} x^{2}+v_{2}\right)+\phi(x) \frac{1}{72 n}\left(v_{3} x^{5}+v_{4} x^{3}+v_{5} x\right) .
$$

Between $Q_{n}$ and $H_{n}$, it holds that

$$
Q_{n}\left(x+\frac{\zeta}{\sqrt{n} \sigma_{1}^{3}}\right)=H_{n}(x)+o\left(n^{-1}\right)
$$

The asymptotic expansion of the statistic $\hat{\sigma}_{n}^{-1} \sqrt{n}\left(Y_{n}-E Y_{n}\right)$ is given by the following.

Lemma 2.3. (Maesono (1996)) Assume that $E\left|g\left(X_{i_{1}}, \ldots, X_{i_{k}}\right)\right|^{2}<\infty$ for $1 \leq i_{1} \leq$ $\cdots \leq i_{k} \leq k, E\left|g\left(X_{1}, X_{2}, \ldots, X_{k}\right)\right|^{9}<\infty, E\left|g\left(X_{1}, X_{1}, X_{2}, \ldots, X_{k}\right)\right|^{4+\varepsilon}<\infty$ for $\varepsilon>0$, and $\left.E\left[g^{(1)}\left(X_{1}\right)\right)\right]^{4}<\infty, \sigma_{1}^{2}>0$. Furthermore we assume that $E\left[\left|a_{1}\left(X_{1}\right)\right|^{3}+\mid a_{3}\left(X_{1}, X_{2}\right.\right.$, $\left.\left.X_{3}\right)\left.\right|^{4}\right]<\infty$ and $\lim \sup _{|t| \rightarrow \infty}\left|E\left[\exp \left\{i t g^{(1)}\left(X_{1}\right)\right\}\right]\right|<1$. If either condition (C) or (D) is satisfied, we have

$$
\sup _{-\infty<x<\infty}\left|P\left(\hat{\sigma}_{n}^{-1} \sqrt{n}\left(Y_{n}-E Y_{n}\right) \leq x\right)-H_{n}(x)\right|=o\left(n^{-1}\right) .
$$




\section{Studentized Y-statistic about $\theta$}

At first, we note that

$$
\hat{\sigma}_{n}^{-1} \sqrt{n}\left(Y_{n}-\theta\right)=\hat{\sigma}_{n}^{-1} \sqrt{n}\left(Y_{n}-E Y_{n}\right)+\hat{\sigma}_{n}^{-1} \sqrt{n}\left(E Y_{n}-\theta\right) .
$$

By Nomachi et al. (2002), (3.5), we have

$$
\sqrt{n}\left(E Y_{n}-\theta\right)=\frac{\mu_{k}}{\sqrt{n}}+O\left(n^{-3 / 2}\right)
$$

where $\mu_{k}=\delta_{k}\left(\theta_{k-1}-\theta\right)$.

If we put $R_{2 n}=o_{p}^{*}\left(n^{-1}\right)$, then $\sqrt{n}\left(E Y_{n}-\theta\right) R_{2 n}=o_{p}^{*}\left(n^{-1}\right)$. Because for a constant $c>0$ we have $P\left(\left|\sqrt{n}\left(E Y_{n}-\theta\right) R_{2 n}\right| \geq c n^{-1}(\log n)^{-1}\right) \leq P\left(\left|R_{2 n}\right| \geq c n^{-1}(\log n)^{-1}\right)=$ $o\left(n^{-1}\right)$, since $\sqrt{n}\left(E Y_{n}-\theta\right) \leq 1$ for a large $n$. We multiply (2.3) by (3.2), and use this fact. Then, we get

$$
k \sigma_{1} \hat{\sigma}_{n}^{-1} \sqrt{n}\left(E Y_{n}-\theta\right)=\frac{\mu_{k}}{\sqrt{n}}\left\{1-\frac{1}{n \sigma_{1}^{2}} \sum_{i=1}^{n} f_{1}\left(X_{i}\right)\right\}+R_{n}^{*}+o_{p}^{*}\left(n^{-1}\right)
$$

where $E\left|R_{n}^{*}\right|=O\left(n^{-3 / 2}\right)$. Thus from (2.4), (3.1) and (3.3) we get

$$
\hat{\sigma}_{n}^{-1} \sqrt{n}\left(Y_{n}-\theta\right)=\frac{\sqrt{n}}{\sigma_{1}} U_{n}^{* *}+\frac{1}{\sqrt{n}}\left\{-\frac{\zeta}{\sigma_{1}^{3}}+\frac{\mu_{k}}{k \sigma_{1}}\right\}+R_{n}^{*}+o_{p}^{*}\left(n^{-1}\right),
$$

where

$$
U_{n}^{* *}=\frac{1}{n} \sum_{i=1}^{n}\left\{g^{(1)}\left(X_{i}\right)+\frac{a_{1}^{*}\left(X_{i}\right)}{n}\right\}+\frac{1}{n^{2}} \sum_{1 \leq i<j \leq n} a_{2}\left(X_{i}, X_{j}\right)+\frac{1}{n^{3}} \sum_{1 \leq i<j<l \leq n} a_{3}\left(X_{i}, X_{j}, X_{l}\right),
$$

and

$$
a_{1}^{*}\left(X_{i}\right)=a_{1}\left(X_{i}\right)-\frac{\mu_{k}}{k \sigma_{1}^{3}} f_{1}\left(X_{i}\right) .
$$

We can also obtain the expansion (3.4) by multiplying (2.3) and the following (3.6). For the detail of this multiplication, see Appendix.

Lemma 3.1. (Yamato et al. (2003)) Assume that $d(k, k)>0$ and $E\left|g\left(X_{i_{1}}, \ldots, X_{i_{k}}\right)\right|^{2}<\infty$ for $1 \leq i_{1} \leq \cdots \leq i_{k} \leq k$. Then, we have

$$
\sqrt{n}\left(Y_{n}-\theta\right)=Y_{n}^{* *}+\frac{\mu_{k}}{\sqrt{n}}+R_{n}^{\prime}
$$

where $E\left|R_{n}^{\prime}\right|^{2}=O\left(n^{-3}\right)$ and

$$
\begin{aligned}
& Y_{n}^{* *}=k\left(1-\frac{\delta_{k}}{n}\right) \frac{1}{n^{1 / 2}} \sum_{i=1}^{n} g^{(1)}\left(X_{i}\right)+(k-1) \delta_{k} \frac{1}{n^{3 / 2}} \sum_{i=1}^{n} g_{(k-1)}^{(1)}\left(X_{i}\right) \\
& +k(k-1) \frac{1}{n^{3 / 2}} \sum_{1 \leq i<j \leq n} g^{(2)}\left(X_{i}, X_{j}\right)+k(k-1)(k-2) \frac{1}{n^{5 / 2}} \sum_{1 \leq i<j<l \leq n} g^{(3)}\left(X_{i}, X_{j}, X_{l}\right) .
\end{aligned}
$$


In the asymptotic evaluation of of $\hat{\sigma}_{n}^{-1} \sqrt{n}\left(Y_{n}-\theta\right)$ with remainder term $o\left(n^{-1}\right)$, we can neglect at first the term $o_{p}^{*}\left(n^{-1}\right)$ of (3.4) by using the relation given by Lemma 5.3 and then the terms $R_{n}^{*}$ of (3.4) by using the relation given by Lemma 5.2. Thus, we can get the following.

Lemma 3.2. Assume that $d(k, k)>0, E\left|g\left(X_{i_{1}}, \ldots, X_{i_{k}}\right)\right|^{2}<\infty$ for $1 \leq i_{1} \leq \cdots \leq$ $i_{k} \leq k, E\left|g\left(X_{1}, X_{2}, X_{3}, \ldots, X_{k}\right)\right|^{9}<\infty$ and $E\left|g\left(X_{1}, X_{1}, X_{2}, \ldots, X_{k}\right)\right|^{4+\varepsilon}<\infty$ for $\varepsilon>0$. Then, we have

$$
\sup _{-\infty<x<\infty}\left|P\left(\hat{\sigma}_{n}^{-1} \sqrt{n}\left(Y_{n}-\theta\right) \leq x\right)-P\left(\frac{\sqrt{n}}{\sigma_{1}} U_{n}^{* *}+\frac{1}{\sqrt{n}}\left\{-\frac{\zeta}{\sigma_{1}^{3}}+\frac{\mu_{k}}{k \sigma_{1}}\right\} \leq x\right)\right|=o\left(n^{-1}\right) .
$$

$U_{n}^{* *}$ is different from $U_{n}^{*}$ only in the term $a_{1}^{*}$. Thus the Edgeworth expansion of $U_{n}^{* *}$ is different from $U_{n}^{*}$ in the term $\lambda_{7}$. By Lemma 2.2, we get the following.

LEMma 3.3. Assume that $d(k, k)>0$. Furthermore, we assume that $E\left|g\left(X_{i_{1}}, \ldots, X_{i_{k}}\right)\right|^{2}<\infty$ for $1 \leq i_{1} \leq \cdots \leq i_{k} \leq k, E\left[g^{(1)}\left(X_{1}\right)\right]^{4}<\infty, \sigma_{1}^{2}>0$, $E\left[\left|a_{1}\left(X_{1}\right)\right|^{3}+\left|a_{3}\left(X_{1}, X_{2}, X_{3}\right)\right|^{4}\right]<\infty$ and $\lim \sup _{|t| \rightarrow \infty}\left|E\left[\exp \left\{i t g^{(1)}\left(X_{1}\right)\right\}\right]\right|<1$. If either condition $(\mathrm{C})$ or $(\mathrm{D})$ is satisfied, we have

$$
\sup _{-\infty<x<\infty}\left|P\left(\sqrt{n} \sigma_{1}^{-1} U_{n}^{* *} \leq x\right)-Q_{n}^{*}(x)\right|=o\left(n^{-1}\right)
$$

where $Q_{n}^{*}(x)$ is obtained from $Q_{n}(x)$ by replacing $\lambda_{7}$ with

$$
\lambda_{7}^{*}=\lambda_{7}-\frac{\mu_{k}}{k \sigma_{1}^{3}}\left(\frac{1}{2} e_{1}+e_{2}\right)
$$

The last term of the above right-hand side is due to the bias of the Y-statistic. We also know that

$$
Q_{n}^{*}(x)=Q_{n}(x)-\frac{\mu_{k}}{n k \sigma_{1}^{5}}\left(\frac{1}{2} e_{1}+e_{2}\right) x \phi(x) .
$$

By (2.6), we have

$$
Q_{n}\left(x+\frac{\zeta}{\sqrt{n} \sigma_{1}^{3}}-\frac{\mu_{k}}{\sqrt{n} k \sigma_{1}}\right)=H_{n}\left(x-\frac{\mu_{k}}{\sqrt{n} k \sigma_{1}}\right)+o\left(n^{-1}\right)
$$

and by Lemma 5.4

$$
\begin{gathered}
H_{n}\left(x-\frac{\mu_{k}}{\sqrt{n} k \sigma_{1}}\right)=\Phi(x)+\phi(x) \frac{1}{6 \sqrt{n}}\left(v_{1} x^{2}+v_{2}-6 \frac{\mu_{k}}{k \sigma_{1}}\right) \\
+\phi(x) \frac{1}{72 n}\left(v_{3} x^{5}+\left(v_{4}+12 \frac{v_{1} \mu_{k}}{k \sigma_{1}}\right) x^{3}+\left[v_{5}+12 \frac{\mu_{k}}{k \sigma_{1}}\left(v_{2}-2 v_{1}\right)\right] x-36\left(\frac{\mu_{k}}{k \sigma_{1}}\right)^{2}\right)+O\left(n^{-3 / 2}\right) .
\end{gathered}
$$

By (3.9), (3.10), (3.11) and Lemma 5.4, we can get

$$
Q_{n}^{*}\left(x+\frac{1}{\sqrt{n}}\left\{\frac{\zeta}{\sigma_{1}^{3}}-\frac{\mu_{k}}{k \sigma_{1}}\right\}\right)=H_{n}^{*}(x)+O\left(n^{-3 / 2}\right),
$$


where

$$
\begin{array}{r}
H_{n}^{*}(x)=\Phi(x)+\phi(x) \frac{1}{6 \sqrt{n}}\left(v_{1} x^{2}+v_{2}-\frac{6 \mu_{k}}{k \sigma_{1}}\right)+\phi(x) \frac{1}{72 n}\left\{v_{3} x^{5}+\left(v_{4}+\frac{v_{1} \mu_{k}}{k \sigma_{1}}\right) x^{3}\right. \\
\left.+\left[v_{5}+12 \frac{\mu_{k}}{k \sigma_{1}}\left(v_{2}-2 v_{1}\right)-72 \frac{\mu_{k}}{k \sigma_{1}^{5}}\left(\frac{1}{2} e_{1}+e_{2}\right)\right] x-36\left(\frac{\mu_{k}}{k \sigma_{1}}\right)^{2}\right\} .
\end{array}
$$

Thus, by (3.7), (3.8), (3.11) and (3.12) we get the following.

Theorem 3.4. Assume that $d(k, k)>0, E\left|g\left(X_{i_{1}}, \ldots, X_{i_{k}}\right)\right|^{2}<\infty$ for $1 \leq i_{1} \leq \cdots \leq i_{k} \leq k, E\left[g^{(1)}\left(X_{1}\right)\right]^{4}<\infty, \sigma_{1}^{2}>0, E\left|g\left(X_{1}, X_{2}, \ldots, X_{k}\right)\right|^{9}<\infty$, and $E\left|g\left(X_{1}, X_{1}, X_{2}, \ldots, X_{k}\right)\right|^{4+\varepsilon}<\infty$ for $\varepsilon>0$. Furthermore we assume that $\limsup _{|t| \rightarrow \infty}\left|E\left[\exp \left\{i t g^{(1)}\left(X_{1}\right)\right\}\right]\right|<1$ and $E\left[\left|a_{1}\left(X_{1}\right)\right|^{3}+\left|a_{3}\left(X_{1}, X_{2}, X_{3}\right)\right|^{4}\right]<\infty$. If either condition $(\mathrm{C})$ or $(\mathrm{D})$ is satisfied, we have

$$
\sup _{-\infty<x<\infty}\left|P\left(\hat{\sigma}_{n}^{-1} \sqrt{n}\left(Y_{n}-\theta\right) \leq x\right)-H_{n}^{*}(x)\right|=o\left(n^{-1}\right) .
$$

As stated after Lemma 2.2, one of the conditions of Theorem $3.4 E\left[\left|a_{1}\left(X_{1}\right)\right|^{3}+\right.$ $\left.\left|a_{3}\left(X_{1}, X_{2}, X_{3}\right)\right|^{4}\right]<\infty$ is satisfied if $E\left|g^{(1)}\left(X_{1}\right)\right|^{12}<\infty, E\left|g^{(2)}\left(X_{1}, X_{2}\right)\right|^{12}<\infty$, and $E\left|g^{(3)}\left(X_{1}, X_{2}, X_{3}\right)\right|^{12}<\infty, E\left|g_{(k-1)}^{(1)}\left(X_{1}\right)\right|^{3}<\infty$.

Corollary 3.5. Especially, let the degree $k$ be 2. Assume that $d(k, k)>0$, $E\left|g\left(X_{1}, X_{1}\right)\right|^{4+\varepsilon}<\infty$ for $\varepsilon>0, E\left[g^{(1)}\left(X_{1}\right)\right]^{4}<\infty, \sigma_{1}^{2}>0$, and $E\left|g\left(X_{1}, X_{2}\right)\right|^{9}<\infty$. Furthermore we assume that $\lim \sup _{|t| \rightarrow \infty}\left|E\left[\exp \left\{i t g^{(1)}\left(X_{1}\right)\right\}\right]\right|<1$ and $E\left[\left|a_{1}\left(X_{1}\right)\right|^{3}+\right.$ $\left.\left|a_{3}\left(X_{1}, X_{2}, X_{3}\right)\right|^{4}\right]<\infty$. If either condition (C) or (D) is satisfied, we have (3.13).

In the case of $k=2$, the condition $E\left[\left|a_{1}\left(X_{1}\right)\right|^{3}+\left|a_{3}\left(X_{1}, X_{2}, X_{3}\right)\right|^{4}\right]<\infty$ is satisfied if $E\left|g^{(1)}\left(X_{1}\right)\right|^{12}<\infty, E\left|g^{(2)}\left(X_{1}, X_{2}\right)\right|^{12}<\infty$, and $E\left|g_{(k-1)}^{(1)}\left(X_{1}\right)\right|^{3}<\infty$.

The difference of the Edgeworth expansions of the studentized Y-statistic about its expectation and $\theta$ is the following.

Corollary 3.6.

$$
\begin{aligned}
H_{n}^{*}(x)=H_{n}(x)-\phi(x) \frac{\mu_{k}}{\sqrt{n} k \sigma_{1}}+\phi(x) \frac{1}{72 n}\left\{\frac{v_{1} \mu_{k}}{k \sigma_{1}} x^{3}\right. \\
\left.\quad+\left[12 \frac{\mu_{k}}{\sqrt{n} k \sigma_{1}}\left(v_{2}-2 v_{1}\right)-72 \frac{\mu_{k}}{\sqrt{n} k \sigma_{1}^{5}}\left(\frac{1}{2} e_{1}+2_{2}\right)\right] x-36\left(\frac{\mu_{k}}{\sqrt{n} k \sigma_{1}}\right)^{2}\right\} .
\end{aligned}
$$

Especially, if $\theta_{k-1}=\theta$ then $H_{n}^{*}(x)=H_{n}(x)$.

The condition $\theta_{k-1}=\theta$ above is equivalent to $E g\left(X_{1}, X_{1}, X_{2}, X_{3}, \ldots, X_{k-1}\right)=$ $E g\left(X_{1}, X_{2}, \ldots, X_{k}\right)$. The difference between the Edgeworth expansions about its expectation and $\theta$ appears at the term related with $\mu_{k}$ which arise from the bias. The value of the difference depends on each Y-statistic. The values of $\mu_{k}$ for V-statistic, S-statistic and LB-statistic are as follows.

$$
\mu_{k}=\delta_{k}\left(\theta_{k-1}-\theta\right), \quad \delta_{k}= \begin{cases}\frac{k(k-1)}{2} & (\mathrm{~V}, \mathrm{~S}-\text { statistic }) \\ k(k-1) & (\mathrm{LB}-\text { statistic })\end{cases}
$$


By Remark 3 of Maesono (1996), $H_{n}(x)$ is equal to the Edgeworth expansion of studentized U-statistic using the jackknife variance estimator. Hence, if $\theta_{k-1}=\theta$, then the Edgeworth expansion of studentized Y-statistic about $\theta$ using the jackknife variance estimator is equal to the one of studentized U-statistic using the jackknife variance estimator. This is also read from (3.1) and (3.2).

\section{Examples}

We give examples of the Edgeworth expansion of the studentized Y-statistic about estimable parameter $\theta$.

Example 4.1 We consider the third central moment $\theta=\int(x-\mu)^{3} d F(x)$, where $\mu$ is the mean of $F$. Its kernel $g\left(x_{1}, x_{2}, x_{3}\right)$ is given by

$$
\frac{1}{3}\left(x_{1}^{3}+x_{2}^{3}+x_{3}^{3}\right)-\frac{1}{2}\left(x_{1}^{2} x_{2}+x_{1}^{2} x_{3}+x_{1} x_{2}^{2}+x_{2}^{2} x_{3}+x_{1} x_{3}^{2}+x_{2} x_{3}^{2}\right)+2 x_{1} x_{2} x_{3} .
$$

For this kernel, we have $g_{(k-1)}\left(x_{1}, x_{2}\right)=g_{(2)}\left(x_{1}, x_{2}\right)=0$ and $g_{(k-2)}\left(x_{1}\right)=g_{(1)}\left(x_{1}\right)=0$ and so $\theta_{k-1}\left(=\theta_{2}\right)=0 \theta_{k-2}\left(=\theta_{1}\right)=0$. Therefore, we have

$$
Y_{n}=\frac{d(k, k)}{D(n, k)}\left(\begin{array}{l}
n \\
k
\end{array}\right) U_{n}=\frac{d(3,3) n^{2}}{6 D(n, 3)} \sum_{j=1}^{n}\left(X_{j}-\bar{X}\right)^{3}
$$

where $\bar{X}=\sum_{j=1}^{n} X_{j} / n$. We assume that the distribution $F$ has a density. We also assume $E|X|^{27}<\infty$ and denote $j$ th moment of $X$ about the origin by $m^{\prime}{ }_{j}(j=2,3, \ldots, 12)$. In order to study the statistical properties of $Y_{n}$, by (4.1) the mean $\mu$ is assumed to be zero, without loss of generality. Thus, in this case $\theta=m_{3}^{\prime}$ and $\mu_{k}=-\delta_{k} m_{3}^{\prime}$. We consider the two cases that the distribution $F$ is symmetric or not.

Example 4.1.1 We assume that the distribution $F$ is symmetric about zero. In this case, by the symmetry $m_{j}^{\prime}=0(j=3,5, \ldots, 11)$ and $\theta=0$. Then, we have

$$
\begin{aligned}
g^{(1)}\left(x_{1}\right) & =\frac{1}{3}\left(x_{1}^{3}-3 x_{1} m_{2}^{\prime}\right), \\
g^{(2)}\left(x_{1}, x_{2}\right) & =\psi_{2}\left(x_{1}, x_{2}\right)-g^{(1)}\left(x_{1}\right)-g^{(1)}\left(x_{2}\right)-\theta \\
& =\frac{1}{2}\left(-x_{1}^{2} x_{2}-x_{1} x_{2}^{2}+x_{1}{m^{\prime}}_{2}+x_{2} m_{2}^{\prime}\right), \\
g^{(3)}\left(x_{1}, x_{2}, x_{3}\right) & =2 x_{1} x_{2} x_{3} .
\end{aligned}
$$

By the computation based on these, we get

$$
\begin{aligned}
& e_{1}=0, \quad e_{2}=0, \\
& e_{3}=\frac{1}{81}\left(m^{\prime}{ }_{12}-12 m^{\prime}{ }_{10} m_{2}^{\prime}+54 m^{\prime}{ }_{8} m^{\prime 2}{ }_{2}-108 m^{\prime}{ }_{6} m^{\prime 3}{ }_{2}+81 m^{\prime}{ }_{4} m_{2}{ }_{2}^{4}\right) \text {, } \\
& e_{4}=\frac{1}{27}\left(-m^{\prime}{ }_{8} m^{\prime}{ }_{4}+3 m^{\prime}{ }_{8} m_{2}^{\prime 2}+7 m^{\prime}{ }_{6} m^{\prime}{ }_{4} m^{\prime}{ }_{2}-21 m^{\prime}{ }_{6} m^{\prime 3}\right. \\
& \left.-15 m_{4}^{\prime 2} m_{2}^{\prime 2}+54 m_{4}^{\prime} m_{2}^{\prime 4}-27 m_{2}^{\prime 6}\right) \text {, } \\
& e_{5}=\frac{1}{9}\left(m^{\prime 3}-7 m^{\prime 2}{ }_{4}^{\prime 2}{ }_{2}^{2}+15 m^{\prime}{ }_{4} m_{2}^{\prime 4}-9 m^{\prime 6}{ }_{2}\right) \text {, } \\
& e_{6}=\frac{4}{27}\left(m^{\prime 3}-9 m^{\prime 2}{ }_{4}^{\prime 2}{ }_{2}^{2}+27 m^{\prime}{ }_{4} m_{2}^{\prime 4}-27 m^{\prime 6}\right) \text {. }
\end{aligned}
$$


Furthermore,

$$
\sigma_{1}^{2}=\frac{1}{9}\left(m_{6}^{\prime}-6 m_{4}^{\prime} m_{2}^{\prime}+9 m_{2}^{\prime 3}\right), \quad \sigma_{2}^{2}=2\left(m_{4}^{\prime} m_{2}^{\prime}-m_{2}^{\prime 3}\right)
$$

Thus we get

$$
\begin{aligned}
& v_{1}=0, \quad v_{2}=0, \quad v_{3}=0, \\
& v_{4}=\frac{6}{\left(m^{\prime}{ }_{6}-6 m^{\prime}{ }_{4} m^{\prime}{ }_{2}+9 m^{\prime}{ }_{2}^{3}\right)^{2}} \\
& \times\left\{m^{\prime}{ }_{12}-12 m^{\prime}{ }_{10} m_{2}^{\prime}-36 m^{\prime}{ }_{8} m_{4}^{\prime}+162 m^{\prime}{ }_{8} m_{2}^{2}-6 m_{6}^{\prime 2}+324 m_{6}^{\prime} m_{4}^{\prime} m_{2}^{\prime}\right. \\
& \left.-972 m_{6}^{\prime} m_{2}^{\prime 3}+102 m^{\prime 3}-1566 m_{4}^{\prime 2} m_{2}^{\prime 2}+4779 m_{4}^{\prime} m_{2}^{\prime 4}-3240 m_{2}^{\prime 6}\right\} \text {, } \\
& v_{5}=\frac{18}{\left(m^{\prime}{ }_{6}-6 m^{\prime}{ }_{4} m^{\prime}{ }_{2}+9 m^{\prime}{ }_{2}^{3}\right)^{2}} \\
& \times\left\{-m_{12}^{\prime}+12 m^{\prime}{ }_{10} m^{\prime}{ }_{2}+12 m^{\prime}{ }_{8} m^{\prime}{ }_{4}-90 m^{\prime}{ }_{8} m_{2}{ }_{2}^{2}+2 m^{\prime 2}{ }_{6}-90 m^{\prime}{ }_{6} m_{4}{ }_{4} m_{2}^{\prime}\right. \\
& \left.+378 m^{\prime}{ }_{6} m^{\prime 3}-18 m^{\prime 3}+270 m^{\prime 2}{ }_{4} m^{\prime 2}{ }_{2}^{2}-945 m^{\prime}{ }_{4} m^{\prime 4}{ }_{2}+486 m^{\prime 6}{ }_{2}\right\}
\end{aligned}
$$

and

$$
E\left[g^{(1)}\left(X_{1}\right) f_{1}\left(X_{1}\right)\right]=\frac{1}{2} e_{1}+e_{2}=0 .
$$

Now we check the condition $(\mathrm{D})$ : We can write $2 g^{(2)}(x, y)=(x+y)\left(m_{2}^{\prime}-x y\right)=-\left(x^{2}+\right.$ $\left.x-m_{2}^{\prime}\right)\left(y^{2}+y-m_{2}^{\prime}\right)+\left(x^{2}-m_{2}^{\prime}\right)\left(y^{2}-m_{2}^{\prime}\right)+x y$. We can also write

$f_{1}(x) g^{(1)}(y)+f_{1}(y) g^{(1)}(x)=\left[f_{1}(x)+g^{(1)}(x)\right]\left[f_{1}(y)+g^{(1)}(y)\right]-f_{1}(x) f_{1}(y)-g^{(1)}(x) g^{(1)}(y)$.

Thus we have $a_{2}(x, y)=\sum_{j=1}^{6} c_{j} h_{j}(x) h_{j}(y)$ where

$$
\begin{gathered}
h_{1}(x)=x^{2}+x-m_{2}^{\prime}, \quad h_{2}(x)=x^{2}-m_{2}^{\prime}, \quad h_{3}(x)=x, \\
h_{4}(x)=f_{1}(x)+g^{(1)}(x), \quad h_{5}(x)=f_{1}(x), \quad h_{6}(x)=g^{(1)}(x),
\end{gathered}
$$

and

$$
c_{1}=-\frac{1}{2}(k-1), \quad c_{2}=\frac{1}{2}(k-1), \quad c_{3}=\frac{1}{2}(k-1), \quad c_{4}=-\frac{1}{\sigma_{1}^{2}}, \quad c_{5}=c_{6}=\frac{1}{\sigma_{1}^{2}} .
$$

In this example we can write $f_{1}$ as follows:

$$
f_{1}(x)=\frac{1}{18}\left[\left(x^{3}-3 x m_{2}^{\prime}\right)^{2}-9 \sigma_{1}^{2}+6\left(m_{4}^{\prime}-3{m^{\prime}}_{2}^{2}\right)\left(m_{2}^{\prime}-x^{2}\right)\right]
$$

Thus for any $s_{1}, \ldots, s_{K}\left(-\infty<s_{1}, \ldots, s_{K}<\infty\right), g^{(1)}(x)+\sum_{j=1}^{K} s_{j} h_{j}(x)$ is a polynomial of degree 6 with respect to $x$. Therefore the distribution of $g^{(1)}(X)+\sum_{j=1}^{K} s_{j} h_{j}(X)$ has the density for any $s_{1}, \ldots, s_{K}\left(-\infty<s_{1}, \ldots, s_{K}<\infty\right)$ and by Lemma 5.1 the condition (D) is satisfied. We note that the check of the condition (D) of the example (1) of 5 of Yamato et al. (2003) is corrected and may be done like as the above. 
Under our assumption, $\mu_{k}=\delta_{k}\left(\theta_{k-1}-\theta\right)=0$ and the Edgeworth expansion of the studentized Y-statistic about estimable parameter $\theta$ is given by

$$
H_{n}^{*}(x)=\Phi(x)+\phi(x) \frac{1}{72 n}\left\{v_{4} x^{3}+v_{5} x\right\} .
$$

That is, there is no difference of the Edgeworth expansions of the studentized Y-statistic about its expectation and $\theta$. Thus, if the distribution $F$ is symmetric then there is no difference among the Edgeworth expansions of the studentized Y-statistic about its expectation and $\theta$. By Remark 3 of Maesono (1996), this expansion $H_{n}^{*}(x)$ is also equal to the Edgeworth expansion of studentized U-statistic using a jackknife variance estimator.

Example 4.1.2 We assume that the distribution $F$ is not symmetric about zero. In this case, $\theta=m_{3}^{\prime}$ and $\mu_{k}=-\delta_{k} m_{3}^{\prime}$. Now, we have

$$
\begin{aligned}
g^{(1)}\left(x_{1}\right) & =\frac{1}{3} x_{1}^{3}-{m^{\prime}}_{2} x_{1}-m_{3}^{\prime}, \\
g^{(2)}\left(x_{1}, x_{2}\right) & =\frac{1}{2}\left(-x_{1}^{2} x_{2}-x_{1} x_{2}^{2}+x_{1}{m^{\prime}}_{2}+x_{2} m_{2}^{\prime}\right), \\
g^{(3)}\left(x_{1}, x_{2}, x_{3}\right) & =2 x_{1} x_{2} x_{3} .
\end{aligned}
$$

Thus, by the same reason as in Example 4.1.1, Condition (D) is satisfied. By the computation based on these functions, we get

$$
\begin{aligned}
& e_{1}=\frac{1}{27}\left(m^{\prime}{ }_{9}-9 m^{\prime}{ }_{7} m^{\prime}{ }_{2}-3 m^{\prime}{ }_{6} m^{\prime}{ }_{3}+27 m^{\prime}{ }_{5} m_{2}{ }_{2}^{2}\right. \\
& \left.+18 m^{\prime}{ }_{4} m_{3}^{\prime} m_{2}^{\prime}+2 m^{\prime 3}-54 m_{3}^{\prime} m_{2}^{\prime 3}\right) \\
& e_{2}=\frac{2}{9}\left(-m^{\prime}{ }_{5} m^{\prime}{ }_{4}+3 m^{\prime}{ }_{5} m_{2}^{\prime 2}+4 m^{\prime}{ }_{4} m^{\prime}{ }_{3} m^{\prime}{ }_{2}-12 m^{\prime}{ }_{3} m^{\prime 3}{ }_{2}\right) \text {, } \\
& e_{3}=\frac{1}{81}\left(m^{\prime}{ }_{12}-12 m^{\prime}{ }_{10} m^{\prime}{ }_{2}-4 m^{\prime}{ }_{9} m^{\prime}{ }_{3}+54 m^{\prime}{ }_{8} m^{\prime 2}{ }_{2}+36 m^{\prime}{ }_{7} m^{\prime}{ }_{3} m^{\prime}{ }_{2}\right. \\
& +6 m^{\prime}{ }_{6} m_{3}^{\prime 2}-108 m_{6}^{\prime} m_{2}^{\prime 3}-108 m^{\prime}{ }_{5} m^{\prime}{ }_{3} m^{\prime 2}{ }_{2}-36 m^{\prime}{ }_{4} m^{\prime 2}{ }_{3} m^{\prime}{ }_{2} \\
& +81 m^{\prime}{ }_{4} m_{2}^{\prime 4}-3 m^{\prime 4}+162 m_{3}^{\prime 2} m_{2}^{\prime 3} \text { ), } \\
& e_{4}=\frac{1}{27}\left(-m^{\prime}{ }_{8} m^{\prime}{ }_{4}+3 m^{\prime}{ }_{8} m^{\prime 2}{ }_{2}-m^{\prime}{ }_{7} m^{\prime}{ }_{5}+4 m^{\prime}{ }_{7} m^{\prime}{ }_{3} m^{\prime}{ }_{2}+7 m^{\prime}{ }_{6} m^{\prime}{ }_{4} m^{\prime}{ }_{2}\right. \\
& -21 m_{6}^{\prime} m_{2}^{\prime 3}+6 m^{\prime 2} m_{2}^{\prime}+4 m_{5}^{\prime} m_{4}^{\prime} m^{\prime}{ }_{3}-45 m^{\prime}{ }_{5} m^{\prime}{ }_{3} m_{2}^{\prime 2}
\end{aligned}
$$

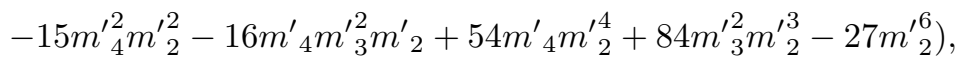

$$
\begin{aligned}
& e_{5}=\frac{1}{9}\left(m^{\prime 2}{ }_{5} m_{2}{ }_{2}+2 m^{\prime}{ }_{5} m^{\prime}{ }_{4} m^{\prime}{ }_{3}-14 m^{\prime}{ }_{5} m^{\prime}{ }_{3} m^{\prime 2}{ }_{2}+m^{\prime}{ }_{4}-7 m^{\prime 2}{ }_{4} m^{\prime 2}\right. \\
& \left.-8 m^{\prime}{ }_{4} m^{\prime 2}{ }_{3} m^{\prime}{ }_{2}+15 m^{\prime}{ }_{4} m^{\prime 4}+40 m^{\prime 2}{ }_{3} m^{\prime 3}{ }_{2}-9 m^{\prime 6}{ }_{2}^{6}\right) \text {, } \\
& e_{6}=\frac{4}{27}\left(m^{\prime 3}-9 m_{4}^{\prime 2} m_{2}^{\prime 2}+27 m^{\prime}{ }_{4} m_{2}^{\prime 4}-27 m_{2}^{\prime 6}\right)
\end{aligned}
$$

Furthermore,

$$
\sigma_{1}^{2}=\frac{1}{9}\left(m_{6}^{\prime}-6 m^{\prime}{ }_{4} m_{2}^{\prime}-m_{3}^{\prime 2}+9 m_{2}^{\prime 3}\right), \quad \sigma_{2}^{2}=2\left(m^{\prime}{ }_{4} m_{2}^{\prime}+m_{3}^{\prime 2}-m_{2}^{\prime 3}\right)
$$

Thus we can get $v_{1}, v_{2}, v_{3}, v_{4}$, and $v_{5}$, which are tedious and we omit to write them. 
We also have

$$
\begin{gathered}
\frac{1}{2} e_{1}+e_{2}=\frac{1}{54}\left(m^{\prime}{ }_{9}-9 m^{\prime}{ }_{7} m^{\prime}{ }_{2}-3 m^{\prime}{ }_{6} m^{\prime}{ }_{3}-12 m^{\prime}{ }_{5} m^{\prime}{ }_{4}+63 m^{\prime}{ }_{5} m_{2}^{\prime 2}\right. \\
\left.+66 m^{\prime}{ }_{4} m^{\prime}{ }_{3} m^{\prime}{ }_{2}+2 m^{\prime}{ }_{3}^{3}-198 m^{\prime}{ }_{3} m^{\prime}{ }_{2}^{3}\right) .
\end{gathered}
$$

The Edgeworth expansion $H_{n}^{*}(x)$ is given by (3.12) with

$$
\mu_{k}= \begin{cases}-\frac{k(k-1)}{2} m_{3}^{\prime} & (\mathrm{V}, \mathrm{S}-\text { statistic }) \\ -k(k-1) m_{3}^{\prime} & (\mathrm{LB}-\text { statistic })\end{cases}
$$

In the relation $(3.14), H_{n}^{*}(x)$ is different from $H_{n}(x)$ with this $\mu_{k}$.

Example 4.2 We consider the kernel $g\left(x_{1}, x_{2}, \ldots, x_{k}\right)=x_{1} x_{2} \cdots x_{k}(k \geq 3)$. This kernel yields estimable parameter $\theta(F)=\mu^{k}$, where $\mu$ is the mean of the distribution $F$. We assume that the distribution $F$ has the density. We also assume that $F$ is symmetric about the mean $\mu(>0)$, and $E|X|^{9}<\infty$ in case of $k=3,4$ and $E\left|X^{2 k+\varepsilon}\right|<\infty$ in case of $k \geq 5$. We shall denote the central moments about the mean by $m_{j}(j=2,4)$. Now, we have

$$
\begin{aligned}
g^{(1)}\left(x_{1}\right) & =\mu^{k-1}\left(x_{1}-\mu\right), \\
g^{(2)}\left(x_{1}, x_{2}\right) & =\mu^{k-2}\left(x_{1}-\mu\right)\left(x_{2}-\mu\right), \\
g^{(3)}\left(x_{1}, x_{2}, x_{3}\right) & =\mu^{k-3}\left(x_{1}-\mu\right)\left(x_{2}-\mu\right)\left(x_{3}-\mu\right) .
\end{aligned}
$$

The computation based on these values yields

$$
\begin{aligned}
& e_{1}=0, \quad e_{2}=(k-1) \mu^{3 k-4} m_{2}^{2}, \quad e_{3}=\mu^{4 k-4} m_{4}, \quad e_{4}=0 \\
& e_{5}=(k-1)^{2} \mu^{4 k-6} m_{2}^{3}, \quad e_{6}=(k-1)(k-2) \mu^{4 k-6} m_{2}^{3} .
\end{aligned}
$$

Furthermore,

$$
\sigma_{1}^{2}=\mu^{2 k-2} m_{2}, \quad \sigma_{2}^{2}=(k-1)^{2} \mu^{2 k-4} m_{2}^{2} .
$$

Thus we get

$$
\begin{aligned}
v_{1} & =\frac{3(k-1) \sqrt{m_{2}}}{\mu}, \quad v_{2}=\frac{3(k-1) \sqrt{m_{2}}}{\mu}, \quad v_{3}=-\frac{9(k-1)^{2} m_{2}}{\mu^{2}}, \\
v_{4} & =6 \xi_{1}^{-4}\left(e_{3}-6 \xi_{1}^{4}+12 e_{4}+6 e_{5}+4 e_{6}\right)-2 \xi_{1}^{-6}\left(2 e_{1}+3 e_{2}\right)\left(2 e_{1}+9 e_{2}\right) \\
& =\frac{6\left\{\mu^{2} m_{4}-6 \mu^{2} m_{2}^{2}+(k-1)(k-5) m_{2}^{3}\right\}}{\mu^{2} m_{2}^{2}}, \\
v_{5} & =3 \xi_{1}^{-6}\left(4 e_{1}^{2}+12 e_{1} e_{2}+3 e_{2}^{2}\right)+18 \xi_{1}^{-4}\left(\xi_{1}^{2} \xi_{2}^{2}-e_{3}+2 \xi_{1}^{4}-4 e_{4}-2 e_{5}\right) \\
& =-\frac{9\left\{2 \mu^{2} m_{4}-4 \mu^{2} m_{2}^{2}+(k-1)(2 k-3) m_{2}^{3}\right\}}{\mu^{2} m_{2}^{2}} .
\end{aligned}
$$

Now we check the condition (D): By the relation derived in Example 4.1, we have $a_{2}(x, y)=\sum_{j=1}^{4} c_{j} h_{j}(x) h_{j}(y)$ where

$$
h_{1}(x)=x-\mu, \quad h_{2}(x)=f_{1}(x)+g^{(1)}(x), \quad h_{3}(x)=f_{1}(x), \quad h_{4}(x)=g^{(1)}(x),
$$


and

$$
c_{1}=-\frac{1}{2} \mu^{k-2}(k-1), \quad c_{2}=-\frac{1}{\sigma_{1}^{2}}, \quad c_{3}=c_{4}=\frac{1}{\sigma_{1}^{2}} .
$$

In this example we can write $f_{1}$ as follows:

$$
f_{1}(x)=\frac{1}{2} \mu^{2 k-3}\left[\mu(x-\mu)^{2}+2(k-1) m_{2}(x-\mu)-\mu m_{2}\right] .
$$

Thus for any $s_{1}, \ldots, s_{K}\left(-\infty<s_{1}, \ldots, s_{K}<\infty\right), g^{(1)}(x)+\sum_{j=1}^{K} s_{j} h_{j}(x)$ is a polynomial of degree 2 with respect to $x$. Therefore the distribution of $g^{(1)}(X)+\sum_{j=1}^{K} s_{j} h_{j}(X)$ has the density for any $s_{1}, \ldots, s_{K}\left(-\infty<s_{1}, \ldots, s_{K}<\infty\right)$ and by Lemma 5.1 the condition (D) is satisfied.

The values of $\mu_{k}$ for V-statistic, S-statistic and LB-statistic are

$$
\mu_{k}= \begin{cases}\frac{k(k-1)}{2}\left(m_{2}^{2}+\mu^{2}-\mu^{3}\right) \mu^{k-3} & (\mathrm{~V}, \mathrm{~S}-\text { statistic }) \\ k(k-1)\left(m_{2}^{2}+\mu^{2}-\mu^{3}\right) \mu^{k-3} & (\mathrm{LB}-\text { statistic })\end{cases}
$$

These values give the difference among the Edgeworth expansions $H_{n}^{*}(x)$. The Edgeworth expansions $H_{n}^{*}(x)$ are given by (3.14) with the above values.

Example 4.3 We consider the kernel

$$
g\left(x_{1}, x_{2}, x_{3}\right)=\frac{1}{3}\left\{I\left(x_{1}>x_{2}+x_{3}\right)+I\left(x_{2}>x_{1}+x_{3}\right)+I\left(x_{3}>x_{1}+x_{2}\right)\right\},
$$

where $I(A)$ is the indicator function of an event $A$. This kernel yields the estimable parameter $\theta(F)=E\left[1-F\left(X_{1}+X_{2}\right)\right]$ which measures the degree to which a life distribution $F$ has the NBU (new better than used) property. If $X_{1}$ and $X_{2}$ are random variables having the life distribution $F$, the NBU property is denoted by $P\left(X_{1}>x\right) \geq$ $P\left(X_{2}>x+y \mid X_{2}>y\right)$ for $x, y>0$. (See, Hollander and Proschan (1972), and Lee (1990)). We note that

$$
\begin{aligned}
\psi_{1}(x) & =\frac{1}{3} E\left[F\left(x-X_{3}\right)\right]+\frac{2}{3} E\left[1-F\left(x+X_{3}\right)\right], \\
\psi_{2}\left(x_{1}, x_{2}\right) & =\frac{1}{3}\left[F\left(\left|x_{2}-x_{1}\right|\right)+1-F\left(x_{1}+x_{2}\right)\right] .
\end{aligned}
$$

For the corresponding U-statistic, we shall derive Edgeworth expansion in cases that $F$ are the uniform distribution $\mathrm{U}(0,1)$ and the exponential distribution $e(1)$ with parameter 1. Since the kernel (4.2) is scale invariant, the Edgeworth expansion for the uniform distribution $\mathrm{U}(0,1)$ is equal to the one for the uniform distribution $\mathrm{U}(0, \alpha), \alpha>0$. The Edgeworth expansion for the exponential distribution $e(1)$ is also equal to the one for the exponential distribution $e(\alpha), \alpha>0$.

Example 4.3.1 We assume that $F$ is the uniform distribution $\mathrm{U}(0,1)$. Then $\theta(F)=$ 
$1 / 6$, and

$$
\begin{aligned}
& g^{(1)}\left(x_{1}\right) \\
& \quad=\frac{1}{2} x_{1}^{2}-\frac{2}{3} x_{1}+\frac{1}{6} \quad(0<x<1), \\
& g^{(2)}\left(x_{1}, x_{2}\right) \\
& \quad= \begin{cases}\frac{1}{3}\left[\left|x_{1}-x_{2}\right|-\left(x_{1}+x_{2}\right)\right]-\frac{1}{2}\left(x_{1}^{2}+x_{2}^{2}\right)+\frac{2}{3}\left(x_{1}+x_{2}\right)-\frac{1}{6} \quad\left(0<x_{1}+x_{2}<1\right) \\
\frac{1}{3}\left|x_{1}-x_{2}\right|-\frac{1}{2}\left(x_{1}^{2}+x_{2}^{2}\right)+\frac{2}{3}\left(x_{1}+x_{2}\right)-\frac{1}{2} \quad\left(x_{1}+x_{2}>1\right) .\end{cases}
\end{aligned}
$$

By using the expression of $g^{(1)}$ and $g^{(2)}$ to

$$
\begin{aligned}
E\left[g^{(1)}\left(X_{2}\right) g^{(2)}\left(X_{1}, X_{2}\right) \mid X_{1}=x_{1}\right]= & \int_{0<x_{2}<1,0<x_{1}+x_{2}<1} g^{(1)}\left(x_{2}\right) g^{(2)}\left(x_{1}, x_{2}\right) d x_{2} \\
& +\int_{0<x_{2}<1, x_{1}+x_{2} \geq 1} g^{(1)}\left(x_{2}\right) g^{(2)}\left(x_{1}, x_{2}\right) d x_{2},
\end{aligned}
$$

we get

$$
\begin{aligned}
& E\left[g^{(1)}\left(X_{2}\right) g^{(2)}\left(X_{1}, X_{2}\right) \mid X_{1}=x_{1}\right] \\
& \quad=\frac{1}{3}\left\{2 x_{1} \int_{0}^{x_{1}} g^{(1)}\left(x_{2}\right) d x_{2}-\int_{0}^{x_{1}} x_{2} g^{(1)}\left(x_{2}\right) d x_{2}+\int_{x_{1}}^{1} x_{2} g^{(1)}\left(x_{2}\right) d x_{2}\right\} \\
& -\int_{0}^{1}\left[g^{(1)}\left(x_{2}\right)\right]^{2} d x_{2}+\frac{1}{3}\left\{\left(1-x_{1}\right) \int_{0}^{1-x_{1}} g^{(1)}\left(x_{2}\right) d x_{2}-\int_{0}^{1-x_{1}} x_{2} g^{(1)}\left(x_{2}\right) d x_{2}\right\} .
\end{aligned}
$$

Thus we get

$$
E\left[g^{(1)}\left(X_{2}\right) g^{(2)}\left(X_{1}, X_{2}\right) \mid X_{1}=x_{1}\right]=\frac{1}{24} x_{1}^{4}-\frac{5}{54} x_{1}^{3}+\frac{1}{18} x_{1}^{2}-\frac{1}{270} .
$$

By the computation based on these functions using Mathematica ver. 4.0, we get

$$
\sigma_{1}^{2}=\frac{1}{270} \doteq 0.0037, \quad \sigma_{2}^{2}=\frac{1}{135} \doteq 0.0074
$$

and

$$
\begin{gathered}
e_{1}=\frac{1}{140} \doteq 0.00714, \quad e_{2} \doteq-\frac{1}{4536}=-0.00022, \quad e_{3}=\frac{1}{280} \doteq 0.00357 \\
e_{4}=-\frac{19}{1360800} \doteq-0.00001, \quad e_{5}=\frac{1}{72900} \doteq 0.000014
\end{gathered}
$$

Since we can write

$$
e_{6}=(k-1)(k-2) E\left[g^{(1)}\left(X_{1}\right) h\left(X_{1}\right)\right]
$$

where $h(x)=\int_{0<y+z<x} g^{(1)}(y) g^{(1)}(z) d y d z$, we have

$$
e_{6}=-\frac{1}{272160} \doteq-0.0000037
$$


Thus we get

$$
\begin{gathered}
v_{1}=\frac{309 \sqrt{30}}{28} \doteq 60.44510, \quad v_{2}=\frac{21 \sqrt{30}}{4} \doteq 28.7554, \quad v_{3}=-\frac{1432215}{292} \doteq-4904.8459, \\
v_{4}=-\frac{4878216261}{960400} \doteq-5079.35887, \quad v_{5}=\frac{2495655}{392} \doteq 6366.4668 .
\end{gathered}
$$

Now we check the condition $(\mathrm{C})$ : We take $h(y)=y^{l}(0<y<1, l=1,2, \ldots)$ for $(L h)(x)=E\left[a_{2}\left(x, X_{2}\right) h\left(X_{2}\right)\right](0<x<1)$. Since $f_{1}$ and $g^{(1)}$ are polynomials of degrees 4 and 2 , respectively, the term related to $f_{1}$ and $g^{(1)}$ in $E\left[a_{2}\left(x, X_{2}\right) h\left(X_{2}\right)\right]$ is a polynomial of degree 4 . Among the terms of $g^{(2)},|x-y|$ yields the integral

$$
\int_{0}^{1}|x-y| y^{l} d y=\frac{2}{(l+1)(l+2)} x^{l+2}-\frac{1}{l+1} x+\frac{1}{l+2} \quad(0<x<1)
$$

which is a polynomial of degree $l+2$. Among the terms of $g^{(2)}$, the other term yields a polynomial of degree 2 . That is, $(L h)(x)(0<x<1)$ is a polynomial of degree $l+2$ for $h(y)=y^{l}(0<y<1, l=2,3, \ldots)$. Thus if we choose $h_{j}(y)=y^{j}$ for $j=2,3, \ldots, K$, then $h_{1}\left(x_{1}\right), \ldots, h_{K}\left(x_{1}\right)$ are linearly independent and the covariance matrix of $\left(h_{1}\left(X_{1}\right), \ldots, h_{K}\left(X_{1}\right)\right)$ is positive definite. Thus the condition $(\mathrm{C})$ is satisfied.

The values of $\mu_{k}$ for V-statistic, S-statistic and LB-statistic are

$$
\mu_{k}=\left\{\begin{array}{cc}
\frac{1}{4} & (\mathrm{~V}, \mathrm{~S}-\text { statistic }) \\
\frac{1}{2} & (\mathrm{LB}-\text { statistic })
\end{array}\right.
$$

These values give the difference among the Edgeworth expansions $H_{n}^{*}(x)$. The Edgeworth expansions $H_{n}^{*}(x)$ are given by (3.14) with the above values.

Example 4.3.2 We assume that $F$ is the exponential distribution $e(1)$. Then $\theta(F)=1 / 4$, and

$$
\begin{aligned}
g^{(1)}\left(x_{1}\right) & =\frac{1}{12}-\frac{1}{3} x_{1} e^{-x_{1}}, \quad\left(x_{1}>0\right) \\
g^{(2)}\left(x_{1}, x_{2}\right) & =-\frac{1}{12}+\frac{1}{3}\left[x_{1} e^{-x_{1}}+x_{2} e^{-x_{2}}-e^{-\left|x_{1}-x_{2}\right|}+e^{-\left(x_{1}+x_{2}\right)}\right] \quad\left(x_{1}, x_{2}>0\right) .
\end{aligned}
$$

By using the expression of $g^{(1)}\left(x_{1}\right)$ and $g^{(2)}\left(x_{1}, x_{2}\right)$ to

$$
\begin{aligned}
E & {\left[g^{(1)}\left(X_{2}\right) g^{(2)}\left(X_{1}, X_{2}\right) \mid X_{1}=x_{1}\right] } \\
& =\int_{0}^{x_{1}} g^{(1)}\left(x_{2}\right) g^{(2)}\left(x_{1}, x_{2}\right) d x_{2}+\int_{x_{1}}^{\infty} g^{(1)}\left(x_{2}\right) g^{(2)}\left(x_{1}, x_{2}\right) d x_{2},
\end{aligned}
$$

we get

$$
E\left[g^{(1)}\left(X_{2}\right) g^{(2)}\left(X_{1}, X_{2}\right) \mid X_{1}=x_{1}\right]=-\frac{5}{3888}-\frac{8}{81} e^{-2 x_{1}}+\frac{8}{81} e^{-x_{1}}-\frac{2}{27} x_{1} e^{-2 x_{1}}-\frac{1}{36} x_{1} e^{-x_{1}} .
$$


By the computation based on these functions using Mathematica ver. 4.0, we get

$$
\sigma_{1}^{2}=\frac{5}{3888} \doteq 0.001286, \quad \sigma_{2}^{2}=\frac{251}{972} \doteq 0.25823
$$

and

$$
\begin{gathered}
e_{1}=\frac{1}{31104} \doteq 0.000032, \quad e_{2}=-\frac{5}{69984} \doteq-0.000071, \quad e_{3}=\frac{2171}{583200000} \doteq 0.0000037 \\
e_{4}=\frac{-10127}{9447840000} \doteq-0.000001, \quad e_{5}=\frac{2083}{157464000} \doteq 0.000013
\end{gathered}
$$

By the method similar to Example 4.3.1, we have

$$
e_{6}=\frac{1}{1119744} \doteq 0.00000089
$$

Thus we get

$$
\begin{gathered}
v_{1}=-\frac{21 \sqrt{3}}{5 \sqrt{5}} \doteq-3.253306, \quad v_{2}=-\frac{51 \sqrt{3}}{10 \sqrt{5}} \doteq-3.95044, \quad v_{3}=-\frac{1323}{125} \doteq-10.584, \\
v_{4}=-\frac{787836542389301}{134369280000000} \doteq-5.86322, \quad v_{5}=\frac{7274727}{78125} \doteq 93.11651 .
\end{gathered}
$$

Now we check the condition $(\mathrm{C})$ : We take $h(y)=e^{-l y}(y>0, l=1,2, \ldots)$ for $(L h)(x)=$ $E\left[a_{2}\left(x, X_{2}\right) h\left(X_{2}\right)\right](x>0)$. The terms related to $f_{1}$ and $g^{(1)}$ in $E\left[a_{2}\left(x, X_{2}\right) h\left(X_{2}\right)\right]$ contain exponential functions $e^{-x}$ or $e^{-2 x}$. Among the terms of $g^{(2)}, e^{-|x-y|}$ yields the integral

$$
\int_{0}^{\infty} e^{-|x-y|} e^{-l y} d y=\frac{1}{(l-1)} e^{-x}-\frac{2}{(l-1)(l+1)} e^{-l x} \quad(x>0)
$$

which contain exponents $e^{-x}$ and $e^{-l x}$. Among the terms of $g^{(2)}$, the other terms are constant or contain a exponential function $e^{-x}$. That is, $(L h)(x)$ contains exponent $e^{-x}$, $e^{-2 x}$ and $e^{-l x}$ for $h(y)=e^{-l y}(l=2,3, \ldots)$. Thus if we choose $h_{j}(y)=e^{-(j+1) y}(y>0)$ for $j=1,2, \ldots, K$, then $h_{1}\left(x_{1}\right), \ldots, h_{K}\left(x_{1}\right)$ are linearly independent and the covariance matrix of $\left(h_{1}\left(X_{1}\right), \ldots, h_{K}\left(X_{1}\right)\right)$ is positive definite. Thus the condition (C) is satisfied.

The values of $\mu_{k}$ for V-statistic, S-statistic and LB-statistic are

$$
\mu_{k}= \begin{cases}-\frac{5}{12} & (\mathrm{~V}, \mathrm{~S}-\text { statistic }) \\ -\frac{5}{6} & (\mathrm{LB}-\text { statistic }) .\end{cases}
$$

These values give the difference among the Edgeworth expansions $H_{n}^{*}(x)$. The Edgeworth expansions $H_{n}^{*}(x)$ are given by (3.14) with the above values.

Next, we consider about the kernel of degree 2 .

Example 4.4 We consider the variance $\theta=\int(x-\mu)^{2} d F(x)$. Its kernel $g\left(x_{1}, x_{2}\right)$ is given by

$$
\frac{1}{2}\left(x_{1}^{2}+x_{2}^{2}-2 x_{1} x_{2}\right) .
$$


For this kernel, we have $g_{(k-1)}\left(x_{1}\right)=g_{(1)}\left(x_{1}\right)=0$ and so $\theta_{k-1}\left(=\theta_{1}\right)=0$. Therefore, we have

$$
Y_{n}=\frac{d(k, k)}{D(n, k)}\left(\begin{array}{l}
n \\
k
\end{array}\right) U_{n}=\frac{d(2,2) n}{2 D(n, 2)} \sum_{j=1}^{n}\left(X_{j}-\bar{X}\right)^{2} .
$$

We assume that the distribution $F$ has a density. We also assume $E|X|^{18}<\infty$ and denote $j$ th moment of $X$ about the origin by $m^{\prime}{ }_{j}(j=2,3, \ldots, 6)$.

In order to study the statistical properties of $Y_{n}$, by (4.3) the mean $\mu$ is assumed to be zero, without loss of generality. Thus, in this case $\theta=m_{2}^{\prime}$ and $\mu_{k}=-\delta_{k} m_{2}^{\prime}$. We consider the two cases that the distribution $F$ is symmetric or not.

Example 4.4.1 We assume that the distribution $F$ is symmetric about zero. In this case, by the symmetry $m_{j}^{\prime}=0(j=3,5,7)$. Then, we have

$$
g^{(1)}\left(x_{1}\right)=\frac{1}{2}\left(x_{1}^{2}-m_{2}^{\prime}\right), \quad g^{(2)}\left(x_{1}, x_{2}\right)=-x_{1} x_{2}, \quad f_{12}\left(x_{1}\right)=0 .
$$

By the computation based on these, we get

$$
\begin{aligned}
e_{1} & =\frac{1}{8}\left(m_{6}^{\prime}-3 m^{\prime}{ }_{4} m_{2}^{\prime}+2 m_{2}^{\prime 3}\right), \\
e_{2} & =0 \\
e_{3} & =\frac{1}{16}\left(m^{\prime}{ }_{8}-4 m^{\prime}{ }_{6} m_{2}^{\prime}+6 m^{\prime}{ }_{4}{m^{\prime}}_{2}^{2}-3 m^{\prime}{ }_{2}^{4}\right), \\
e_{4} & =0, \quad e_{5}=0, \quad e_{6}=0 .
\end{aligned}
$$

Furthermore,

$$
\sigma_{1}^{2}=\frac{1}{4}\left(m_{4}^{\prime}-m_{2}^{\prime 2}\right), \quad \sigma_{2}^{2}=m_{2}^{\prime 2}
$$

Thus we get

$$
\begin{aligned}
& v_{1}=\frac{2}{\left(m_{4}^{\prime}-m_{2}^{\prime 2}\right)^{3 / 2}}\left\{m_{6}^{\prime}-3 m_{4}^{\prime} m_{2}^{\prime}+2 m_{2}^{\prime 3}\right\}, \\
& v_{2}=\frac{1}{\left(m^{\prime}{ }_{4}-m^{\prime 2}\right)^{3 / 2}}\left\{m_{6}^{\prime}-3 m^{\prime}{ }_{4} m_{2}^{\prime}+2{m^{\prime}}_{2}^{3}\right\} \text {, } \\
& v_{3}=-\frac{4}{\left(m^{\prime}{ }_{4}-m^{\prime 2}{ }_{2}\right)^{3}}\left\{m_{6}^{\prime}-3 m_{4}^{\prime} m_{2}^{\prime}+2 m^{\prime 3}\right\}^{2} \text {, } \\
& v_{4}=\frac{2}{\left(m^{\prime}{ }_{4}-m^{\prime 2}\right)^{3}}\left\{3 m_{8}^{\prime} m_{4}^{\prime}-3 m_{8}^{\prime} m_{2}^{\prime 2}-4 m_{6}^{\prime 2}+12 m_{6}^{\prime} m_{4}^{\prime} m_{2}^{\prime}-4 m_{6}^{\prime} m_{2}^{\prime 3}\right. \\
& \left.-18 m^{\prime 3}+36 m^{\prime 2}{ }_{4} m_{2}^{\prime 2}-33 m^{\prime}{ }_{4} m_{2}^{4}+11 m^{\prime 6}{ }_{2}\right\}, \\
& v_{5}=\frac{3}{\left(m^{\prime}{ }_{4}-m^{\prime 2}{ }_{2}\right)^{3}}\left\{-6 m^{\prime}{ }_{8} m^{\prime}{ }_{4}+6 m^{\prime}{ }_{8}{m^{\prime}}_{2}^{2}+4 m_{6}^{\prime 2}-8 m_{6}^{\prime}{ }_{6}{ }_{2}^{3}\right. \\
& \left.+12 m^{\prime 3}-12 m^{\prime 2}{ }_{4}^{\prime 2}{ }_{2}^{2}-6 m^{\prime}{ }_{4} m_{2}^{\prime 4}+10 m^{\prime 6}{ }_{2}\right\}
\end{aligned}
$$

and

$$
\frac{1}{2} e_{1}+e_{2}=\frac{1}{16}\left(m_{6}^{\prime}-3 m_{4}^{\prime} m_{2}^{\prime}+2 m_{2}^{\prime 3}\right) .
$$


Now we check the condition (D): Since $g^{(2)}\left(x_{1}, x_{2}\right)=-x_{1} x_{2}$, by the same reason stated at the Example 4.1 .1 we have $a_{2}(x, y)=\sum_{j=1}^{6} c_{j} h_{j}(x) h_{j}(y)$ where

$$
h_{1}(x)=x, \quad h_{2}(x)=f_{1}(x)+g^{(1)}(x), \quad h_{3}(x)=f_{1}(x), \quad h_{4}(x)=g^{(1)}(x),
$$

and

$$
c_{1}=-1, \quad c_{2}=-\frac{1}{\sigma_{1}^{2}}, \quad c_{3}=c_{4}=\frac{1}{\sigma_{1}^{2}} .
$$

In this example we can write $f_{1}$ as follows:

$$
f_{1}(x)=\frac{1}{8}\left\{\left(x^{2}-m_{2}^{\prime}\right)^{2}-\left(m_{4}^{\prime}-m_{2}^{\prime 2}\right)\right\}
$$

Thus for any $s_{1}, \ldots, s_{K}\left(-\infty<s_{1}, \ldots, s_{K}<\infty\right), g^{(1)}(x)+\sum_{j=1}^{K} s_{j} h_{j}(x)$ is a polynomial of degree 4 with respect to $x$. Therefore the distribution of $g^{(1)}(X)+\sum_{j=1}^{K} s_{j} h_{j}(X)$ has the density for any $s_{1}, \ldots, s_{K}\left(-\infty<s_{1}, \ldots, s_{K}<\infty\right)$ and by Lemma 5.1 the condition (D) is satisfied.

Example 4.4.2 We assume that the distribution $F$ is not symmetric about zero. Then, we have

$$
g^{(1)}\left(x_{1}\right)=\frac{1}{2}\left(x_{1}^{2}-m^{\prime}{ }_{2}\right), \quad g^{(2)}\left(x_{1}, x_{2}\right)=-x_{1} x_{2}, \quad f_{12}\left(x_{1}\right)=-\frac{1}{2} m_{3}^{\prime} x_{1}
$$

By the computation based on these, we get

$$
\begin{aligned}
& e_{1}=\frac{1}{8}\left(m^{\prime}{ }_{6}-3 m^{\prime}{ }_{4} m^{\prime}{ }_{2}+2 m^{\prime 3}{ }_{2}\right) \text {, } \\
& e_{2}=-\frac{1}{4} m^{\prime 2} \text {, } \\
& e_{3}=\frac{1}{16}\left(m^{\prime}{ }_{8}-4 m^{\prime}{ }_{6} m_{2}^{\prime}+6 m^{\prime}{ }_{4} m_{2}^{\prime 2}-3 m^{\prime 4}{ }_{2}\right) \text {, } \\
& e_{4}=\frac{1}{8}\left(-m^{\prime}{ }_{5} m^{\prime}{ }_{3}+2 m^{\prime 2}{ }_{3} m_{2}^{\prime}\right) \text {, } \\
& e_{5}=\frac{1}{4} m^{\prime 2}{ }_{3}^{\prime}{ }_{2}, \quad e_{6}=0 \text {. }
\end{aligned}
$$

Furthermore

$$
\sigma_{1}^{2}=\frac{1}{4}\left(m_{4}^{\prime}-m_{2}^{\prime 2}\right), \quad \sigma_{2}^{2}=m_{2}^{\prime 2}
$$


Thus we get

$$
\begin{aligned}
& v_{1}=\frac{2}{\left(m^{\prime}{ }_{4}-m^{\prime 2}\right)^{3 / 2}}\left\{m_{6}^{\prime}-3 m_{4}^{\prime} m_{2}^{\prime}-3 m_{3}^{\prime 2}+2 m_{2}^{\prime 3}\right\}, \\
& v_{2}=\frac{1}{\left(m^{\prime}{ }_{4}-m_{2}^{\prime 2}\right)^{3 / 2}}\left\{m_{6}^{\prime}-3 m_{4}^{\prime} m_{2}^{\prime}-6 m_{3}^{\prime 2}+2 m_{2}^{\prime 3}\right\} \text {, } \\
& v_{3}=-\frac{4}{\left(m^{\prime}{ }_{4}-m_{2}^{\prime 2}\right)^{3}}\left\{m_{6}^{\prime}-3 m_{4}^{\prime} m_{2}^{\prime}-3{m^{\prime}}_{3}^{2}+2 m_{2}^{\prime 3}\right\}^{2} \text {, } \\
& v_{4}=\frac{2}{\left(m^{\prime}{ }_{4}-m_{2}^{\prime 2}\right)^{3}} \\
& \times\left\{3 m^{\prime}{ }_{8} m^{\prime}{ }_{4}-3 m^{\prime}{ }_{8} m^{\prime 2}{ }_{2}-4 m^{\prime 2}{ }_{6}+12 m^{\prime}{ }_{6} m^{\prime}{ }_{4} m^{\prime}{ }_{2}+48 m^{\prime}{ }_{6} m^{\prime 2}{ }_{3}-4 m^{\prime}{ }_{6} m^{\prime 3}\right. \\
& -72 m^{\prime}{ }_{5} m^{\prime}{ }_{4} m^{\prime}{ }_{3}+72 m^{\prime}{ }_{5} m^{\prime}{ }_{3} m^{\prime 2}{ }_{2}-18 m^{\prime 3}+36 m^{\prime 2}{ }_{4}{ }^{\prime 2}{ }_{2}+72 m^{\prime}{ }_{4} m^{\prime 2}{ }_{3} m_{2}^{\prime} \\
& \left.-33 m_{4}^{\prime} m_{2}^{\prime 4}-108 m^{\prime 4}-120 m^{\prime 2}{ }_{3}^{\prime 2}{ }_{2}^{3}+11 m_{2}^{\prime 6}\right\} \text {, } \\
& v_{5}=\frac{3}{\left(m_{4}^{\prime}-m_{2}^{\prime 2}\right)^{3}} \\
& \times\left\{-6 m^{\prime}{ }_{8} m^{\prime}{ }_{4}+6 m^{\prime}{ }_{8} m^{\prime 2}{ }_{2}+4 m^{\prime 2}{ }_{6}-24 m^{\prime}{ }_{6} m^{\prime 2}{ }_{3}-8 m^{\prime}{ }_{6} m^{\prime 3}{ }_{2}+48 m^{\prime}{ }_{5} m_{4}^{\prime} m^{\prime}{ }_{3}\right. \\
& -48 m^{\prime}{ }_{5} m^{\prime}{ }_{3} m_{2}^{\prime 2}+12 m^{\prime 3}-12{m^{\prime}}_{4}^{2} m_{2}^{\prime 2}-72 m^{\prime}{ }_{4} m^{\prime 2}{ }_{3} m^{\prime}{ }_{2}-6 m^{\prime}{ }_{4} m_{2}^{\prime 4} \\
& \left.+12{m^{\prime}}_{3}^{4}+96{m^{\prime}}_{3}^{2} m_{2}^{\prime 3}+10 m_{2}^{\prime 6}\right\}
\end{aligned}
$$

and

$$
\frac{1}{2} e_{1}+e_{2}=\frac{1}{16}\left(m_{6}^{\prime}-3 m_{4}^{\prime} m_{2}^{\prime}-4 m^{\prime 2}+2 m_{2}^{\prime 3}\right)
$$

Now we check the condition (D): Since

$$
f_{1}(x)=\frac{1}{8}\left\{\left(x^{2}-m^{\prime}\right)^{2}-\left(m_{4}^{\prime}-m_{2}^{\prime 2}\right)\right\}-\frac{1}{2} m_{3}^{\prime} x
$$

by the same reason as Example 4.4.1, the condition (D) is satisfied.

Example 4.5 We consider the kernel $g\left(x_{1}, x_{2}\right)=x_{1} x_{2}$. This kernel yields estimable parameter $\theta(F)=\mu^{2}$. We assume that the distribution $F$ has the density. We also assume that $F$ is symmetric about the mean $\mu(>0)$ and $E X^{9}<\infty$. The values $e_{2}, e_{3}$ and $e_{5}$ are given by putting $k=2$ in Example 4.2 and, $e_{1}=e_{4}=e_{6}=0$.

Example 4.6 We consider the kernel

$$
g\left(x_{1}, x_{2}\right)=I\left(x_{1}+x_{2}>0\right)
$$

which appears in the Wilcoxon one-sample statistic. We assume that the distribution $F$ has the density and symmetric about zero. Then the value of the estimable parameter $\theta$ is equal to $E\left[I\left(X_{1}+X_{2}>0\right)\right]=1 / 2$. We have also

$$
g_{(1)}\left(x_{1}\right)=I\left(x_{1}>0\right), \quad \theta_{1}\left(=\theta_{k-1}\right)=E I\left(X_{1}>0\right)=\frac{1}{2} .
$$


Therefore $\mu_{2}\left(=\mu_{k}\right)=0$. We note that $1-F(-x)=F(x)$ and $F(X)$ has the uniform distribution $\mathrm{U}(0,1)$. We have

$$
g^{(1)}\left(x_{1}\right)=F\left(x_{1}\right)-\frac{1}{2}, \quad g^{(2)}\left(x_{1}, x_{2}\right)=I\left(x_{1}+x_{2}>0\right)-F\left(x_{1}\right)-F\left(x_{2}\right)+\frac{1}{2},
$$

and

$$
f_{1}\left(x_{1}\right)=0, \quad f_{12}\left(x_{1}\right)=\frac{1}{2}\left\{F\left(x_{1}\right)-F^{2}\left(x_{1}\right)-\frac{1}{6}\right\},
$$

where we use the relation $E\left[F\left(X_{2}\right) I\left(x_{1}+X_{2}>0\right)\right]=\int_{-x_{1}}^{\infty} F\left(x_{2}\right) d F\left(x_{2}\right)=[1-$ $\left.F^{2}\left(-x_{1}\right)\right] / 2=\left[2 F\left(x_{1}\right)-F^{2}\left(x_{1}\right)\right] / 2$. Furthermore, using the relation $E\left[F\left(X_{2}\right) I\left(X_{1}+\right.\right.$ $\left.\left.X_{2}>0\right)\right]=E\left[2 F\left(X_{1}\right)-F^{2}\left(X_{1}\right)\right] / 2=1 / 3$, we get

$$
\sigma_{1}^{2}=\frac{1}{12}, \quad \sigma_{2}^{2}=\frac{1}{12}
$$

Thus we get

$$
e_{1}=e_{2}=0, \quad e_{3}=\frac{1}{80}, \quad e_{4}=-\frac{1}{360}, \quad e_{5}=\frac{1}{720}, \quad e_{6}=0
$$

and

$$
v_{1}=v_{2}=v_{3}=0, \quad v_{4}=-\frac{234}{5}, \quad v_{5}=\frac{216}{5} .
$$

Now we check the condition (C): We assume $E\left|g^{(2)}\right|^{r}<\infty(r>2)$ and take $K$ such that $K>8(4 r-5) /(r-2)$. We take $h(y)=y^{l}(l=1,2, \ldots, K)$ for $(L h)(x)=$ $E\left[a_{2}\left(x, X_{2}\right) h\left(X_{2}\right)\right]$. Under the condition that $F$ has the $K$-th moment, $\int_{-x}^{\infty} y^{l} d F(y)$, $l=1, \ldots, K$, are linearly independent. Since $a_{2}(x, y)=g^{(2)}\left(x_{1}, x_{2}\right)=I\left(x_{1}+x_{2}>\right.$ $0)-F\left(x_{1}\right)-F\left(x_{2}\right)+\frac{1}{2}$, under the same condition, $\left(L h_{1}\right)\left(x_{1}\right), \ldots,\left(L h_{K}\right)\left(x_{1}\right)$ are linearly independent and the covariance matrix of $\left(L h_{1}\right)\left(X_{1}\right), \ldots,\left(L h_{K}\right)\left(X_{1}\right)$ is positive definite, where $h_{l}(y)=y^{l}, l=1, \ldots, K$.

Example 4.7 We consider the kernel

$$
g\left(x_{1}, x_{2}\right)=\frac{1}{2} \max \left(x_{1}, x_{2}\right)=\frac{1}{2}\left[x_{1} I\left(x_{1} \geq x_{2}\right)+x_{2} I\left(x_{1}<x_{2}\right)\right],
$$

which gives the probability weighted moment

$$
\theta=\beta_{1}=\frac{1}{2} E\left[\max \left(X_{1}, X_{2}\right)\right]=E[X F(X)] .
$$

We assume that the distribution $F$ has the uniform distribution $\mathrm{U}(0,1)$. Then we have $\beta_{1}=1 / 3, g_{(1)}\left(x_{1}\right)=x_{1} / 2$, and $\theta_{1}=1 / 4$. Furthermore, we have

$$
\begin{gathered}
2 \psi_{1}\left(x_{1}\right)=E\left[x_{1} I\left(x_{1} \geq X_{2}\right)+X_{2} I\left(x_{1}<X_{2}\right)\right]=\frac{1}{4}\left(1+x_{1}^{2}\right), \\
g^{(1)}\left(x_{1}\right)=\frac{1}{4} x_{1}^{2}-\frac{1}{12} \\
g^{(2)}\left(x_{1}, x_{2}\right)=\frac{1}{2} \max \left(x_{1}, x_{2}\right)-\frac{1}{4}\left(x_{1}^{2}+x_{2}^{2}\right)-\frac{1}{6}
\end{gathered}
$$


and

$$
f_{1}\left(x_{1}\right)=\frac{1}{24} x_{1}^{4}-\frac{1}{24} x_{1}^{2}+\frac{1}{180}, \quad f_{12}\left(x_{1}\right)=\frac{1}{96} x_{1}^{4}-\frac{1}{48} x_{1}^{2}+\frac{7}{1440} .
$$

Therefore we have

Thus we get

$$
\sigma_{1}^{2}=\frac{1}{180}, \quad \sigma_{2}^{2}=\frac{1}{360} .
$$

$$
e_{1}=\frac{1}{3780}, \quad e_{2}=-\frac{1}{3780}, \quad e_{3}=\frac{1}{15120}, \quad e_{4}=-\frac{1}{113400}, \quad e_{5}=\frac{1}{75600}, \quad e_{6}=0
$$

and

$$
v_{1}=-\frac{2 \sqrt{5}}{7}, \quad v_{2}=-\frac{4 \sqrt{5}}{7}, \quad v_{3}=-\frac{20}{49}, \quad v_{4}=-34, \quad v_{5}=\frac{267}{49} \doteq 5.45 .
$$

Now we check the condition $(\mathrm{C})$ : We take $h(y)=y^{l+1}(l=1,2, \ldots, K)$ for $(L h)(x)=$ $E\left[a_{2}\left(x, X_{2}\right) h\left(X_{2}\right)\right]$ and a suitably large $K(>56)$. Since $\int_{0}^{1} y^{l+1} \max (x, y) d y$ is a polynomial of degree $l+3$ in $x,\left(L h_{1}\right)\left(x_{1}\right), \ldots,\left(L h_{K}\right)\left(x_{1}\right)$ are linearly independent. Hence the covariance matrix of $\left(L h_{1}\right)\left(X_{1}\right), \ldots,\left(L h_{K}\right)\left(X_{1}\right)$ is positive definite.

\section{Appendix}

About the condition (D) by Lai and Wang (1993) for Edgeworth expansion, we give a sufficient condition.

LEMma 5.1. We assume that the distribution of $g^{(1)}(X)+\sum_{j=1}^{K} s_{j} h_{j}(X)$ has the density for any $s_{1}, \ldots, s_{K}\left(-\infty<s_{1}, \ldots, s_{K}<\infty\right)$. Then, the relation (2.5) holds, provided the assumptions of Condition (D) preceding (2.5).

Proof. Since the distribution of $g^{(1)}(X)+\sum_{j=1}^{K} s_{j} h_{j}(X)$ has the density for any $s_{1}, \ldots, s_{K}$, we get

$$
E \exp \left(i t\left[g^{(1)}(X)+\sum_{j=1}^{K} s_{j} h_{j}(X)\right]\right) \rightarrow 0 \quad \text { as } \quad|t| \rightarrow \infty \quad \text { for }-\infty<s_{1}, \ldots, s_{K}<\infty .
$$

Thus,

$$
\sup _{\left|s_{1}\right|+\cdots+\left|s_{K}\right| \leq 1}\left|E \exp \left(i t\left[g^{(1)}(X)+\sum_{j=1}^{K} s_{j} h_{j}(X)\right]\right)\right| \rightarrow 0 \quad \text { as } \quad|t| \rightarrow \infty .
$$

On the other hand, for a sufficiently large $|t|$ satisfying $|t|^{-\varepsilon}<1$ with $\varepsilon$ given in Condition (D),

$$
\begin{aligned}
0 & \leq \sup _{\left|s_{1}\right|+\cdots+\left|s_{K}\right| \leq|t|-\varepsilon}\left|E \exp \left(i t\left[g^{(1)}(X)+\sum_{j=1}^{K} s_{j} h_{j}(X)\right]\right)\right| \\
& \leq \sup _{\left|s_{1}\right|+\cdots+\left|s_{K}\right| \leq 1}\left|E \exp \left(i t\left[g^{(1)}(X)+\sum_{j=1}^{K} s_{j} h_{j}(X)\right]\right)\right|,
\end{aligned}
$$

which converges to 0 as $|t| \rightarrow \infty$, because of the previous reason. Thus, (2.5) holds. 
From the proof of Lemma 1.3 in p. 261 of Shorack (2000), we have the following.

Lemma 5.2. (Shorack (2000)) For any random variables $W$ and $\Delta$, it holds that

$$
\sup _{x}|P(W+\Delta \leq x)-P(W \leq x)| \leq 4(E|W \Delta|+E|\Delta|) .
$$

Lemma 1.7 of Petrov (1994) yields the following.

Lemma 5.3. (Lemma 3 of Maesono (1996)) Let $H$ be a bounded function and $\delta$ be a positive constant. For any random variables $W$ and $\Delta$, it holds that

$$
\begin{aligned}
\sup _{x}|P(W+\Delta \leq x)-H(x)| \leq \sup _{x}|P(W \leq x)-H(x)| & +P(|\Delta| \geq \delta) \\
& +\sup _{x}|H(x+\delta)-H(x)| .
\end{aligned}
$$

By the Taylor expansion, we can get the following lemma.

Lemma 5.4. For a positive constant $c$, the following relations hold uniformly with respect to $x \in(-\infty, \infty)$.

$$
\begin{aligned}
& \Phi\left(x-\frac{c}{\sqrt{n}}\right)= \Phi(x)-\frac{c}{\sqrt{n}} \phi(x)-\frac{c^{2}}{2 n} x \phi(x)+O\left(\frac{1}{n^{3 / 2}}\right), \\
& \phi\left(x-\frac{c}{\sqrt{n}}\right)=\phi(x)+\frac{c}{\sqrt{n}} x \phi(x)+\frac{c^{2}}{2 n}\left(x^{2}-1\right) \phi(x)+O\left(\frac{1}{n^{3 / 2}}\right), \\
&\left(x-\frac{c}{\sqrt{n}}\right) \phi\left(x-\frac{c}{\sqrt{n}}\right)= x \phi(x)+\frac{c}{\sqrt{n}}\left(x^{2}-1\right) \phi(x)+\frac{c^{2}}{2 n}\left(x^{3}-3 x\right) \phi(x)+O\left(\frac{1}{n^{3 / 2}}\right), \\
&\left(x-\frac{c}{\sqrt{n}}\right)^{2} \phi\left(x-\frac{c}{\sqrt{n}}\right)= x^{2} \phi(x)+\frac{c}{\sqrt{n}}\left(x^{3}-2 x\right) \phi(x)+\frac{c^{2}}{2 n}\left(x^{4}-5 x^{2}+2\right) \phi(x) \\
&+O\left(\frac{1}{n^{3 / 2}}\right), \\
&\left(x-\frac{c}{\sqrt{n}}\right)^{3} \phi\left(x-\frac{c}{\sqrt{n}}\right)= x^{3} \phi(x)+\frac{c}{\sqrt{n}}\left(x^{4}-3 x^{2}\right) \phi(x)+\frac{c^{2}}{2 n}\left(x^{5}-7 x^{3}+6 x\right) \phi(x) \\
&+O\left(\frac{1}{n^{3 / 2}}\right), \\
&\left(x-\frac{c}{\sqrt{n}}\right)^{5} \phi\left(x-\frac{c}{\sqrt{n}}\right)= x^{5} \phi(x)+\frac{c}{\sqrt{n}}\left(x^{6}-5 x^{4}\right) \phi(x)+\frac{c^{2}}{2 n}\left(x^{7}-11 x^{5}+20 x^{3}\right) \phi(x) \\
&+O\left(\frac{1}{n^{3 / 2}}\right) .
\end{aligned}
$$

Multiplication of (2.3) and (3.6). The term associated with $\mu_{k}$ of (3.4) is obtained by multiplying the second term of (3.6) and the first term of (2.3). The last term of $a_{1}^{*}$ is obtained by multiplying the second terms of (3.6) and (2.3). The terms of the expansion associated with $a_{2}(x, y), a_{3}(x, y, z)$ are obtained directly by multiplication of the right-hand sides of (2.3) and (3.6). Next we consider the first three terms of $a_{1}(x)$, which are

$$
\frac{\delta_{k}}{k}\left[(k-1) g_{(k-1)}^{(1)}(x)-k g^{(1)}(x)\right], \quad \tau g^{(1)}(x), \quad-\frac{1}{\sigma_{1}^{2}}\left[f_{1}(x) g^{(1)}(x)-\zeta\right] .
$$


The terms of the expansion associated with these are also obtained by the same method as the above. Since the third term of $a_{1}$ is subtracted the constant $\zeta$, consequently the constant term associated with $\zeta$ appears in the second term of (3.4).

Now we consider the terms associated with the last two terms of $a_{1}(x)$ which are

$$
-\frac{1}{\sigma_{1}^{2}}\left(E\left[f_{2}\left(x, X_{2}\right) g^{(1)}\left(X_{2}\right)\right]-\frac{3 \zeta}{\sigma_{1}^{2}} f_{1}(x)\right), \quad-\frac{1}{\sigma_{1}^{2}}(k-1) E\left[g^{(2)}\left(x, X_{2}\right) f_{1}\left(X_{2}\right)\right] .
$$

These are obtained by taking the first terms of $\mathrm{H}$-decompositions of

$$
\frac{1}{n^{5 / 2}} \sum_{i<j}\left[g^{(1)}\left(X_{i}\right)+g^{(1)}\left(X_{j}\right)\right]\left[f_{2}\left(X_{i}, X_{j}\right)-\frac{3}{\sigma_{1}^{2}} f_{1}\left(X_{i}\right) f_{1}\left(X_{j}\right)\right]
$$

and

$$
\frac{1}{n^{5 / 2}} \sum_{i<j} g^{(2)}\left(X_{i}, X_{j}\right)\left[f_{1}\left(X_{i}\right)+f_{1}\left(X_{j}\right)\right]
$$

respectively. These (5.1) and (5.2) are obtained directly by multiplication of the righthand sides of (2.3) and (3.6).

\section{Acknowledgement}

The authors would like to express their thanks to the referee for his kind comments.

\section{References}

Helmers, R. (1991). On the Edgeworth expansion and the bootstrap approximation for a studentized U-statistic, Ann. Statist., 19, 470-484.

Hollander, M. and Proschan, F. (1972). Testing whether new is better than used, Ann. Math. Statist., 43, 1136-1146.

Lai, T. L. and Wang, J. Q. (1993). Edgeworth Expansions for symmetric statistics with applications to bootstrap methods, Statistica Sinica, 3, 517-542.

Lee, A. J. (1990). U-statistics, Marcel Dekker, New York.

Maesono, Y. (1995)). On the normal approximations of studentized U-statistics, J. Japan Statist. Soc., 25, No. 1, 19-33.

Maesono, Y. (1996). An Edgeworth expansion of a linear combination of U-statistics, J. Japan Statist. Soc., 26, No. 2, 189-207.

Maesono, Y. (1997). Edgeworth expansions of a studentized U-statistic and a jackknife estimator of variance, J. Statist. Plan. and Inf., 61, 61-84.

Nomachi, T., Kondo, M. and Yamato, H. (2002). Higher order efficiency of linear combinations of U-statistics as estimators of estimable parameters, Scientiae Mathematicae Japonicae, 56, 95-106.

Petrov, V.V. (1994). Limit theorems of probability theory, Oxford Science Publications.

Sen, P. K. (1977). Some invariance principles relating to jackknifing and their role in sequential analysis, Ann. Statist., 5, 316-329. 
Shorack, G. R. (2000). Probability for statisticians, Springer.

Toda, K. and Yamato, H. (2001). Berry-Esseen bounds for some statistics including LB-statistic and V-statistic, J. Japan Statist. Soc., 31, No. 2, 225-237.

Yamato, H., Nomachi, T. and Toda, K. (2003). Edgeworth expansions of some statistics including the LB-statistic and V-statistic, J. Japan Statist. Soc., 33, No. 1, 77-94.

Received October 6, 2003

Revised May 8, 2004 